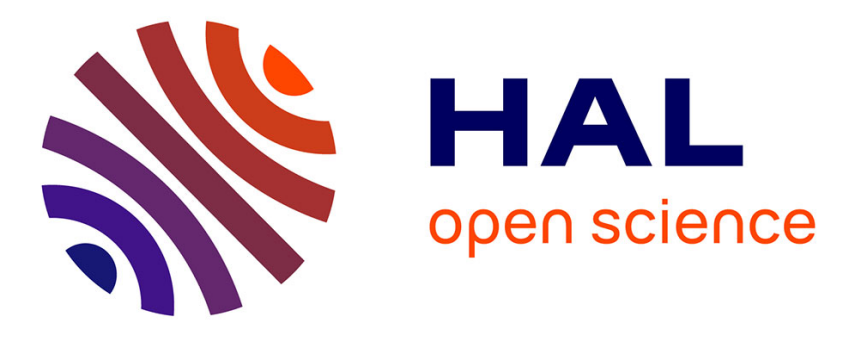

\title{
Strong-field control of electron localization during molecular dissociation
}

Matthias F Kling, Christian Siedschlag, Irina Znakovskaya, Aart Jan Verhoef, Sergey Zherebtsov, Ferenc Krausz, Matthias Lezius, Marc Jj Vrakking

\section{- To cite this version:}

Matthias F Kling, Christian Siedschlag, Irina Znakovskaya, Aart Jan Verhoef, Sergey Zherebtsov, et al.. Strong-field control of electron localization during molecular dissociation. Molecular Physics, 2008, 106 (02-04), pp.455-465. 10.1080/00268970701864739 . hal-00513172

\author{
HAL Id: hal-00513172 \\ https://hal.science/hal-00513172
}

Submitted on 1 Sep 2010

HAL is a multi-disciplinary open access archive for the deposit and dissemination of scientific research documents, whether they are published or not. The documents may come from teaching and research institutions in France or abroad, or from public or private research centers.
L'archive ouverte pluridisciplinaire HAL, est destinée au dépôt et à la diffusion de documents scientifiques de niveau recherche, publiés ou non, émanant des établissements d'enseignement et de recherche français ou étrangers, des laboratoires publics ou privés. 


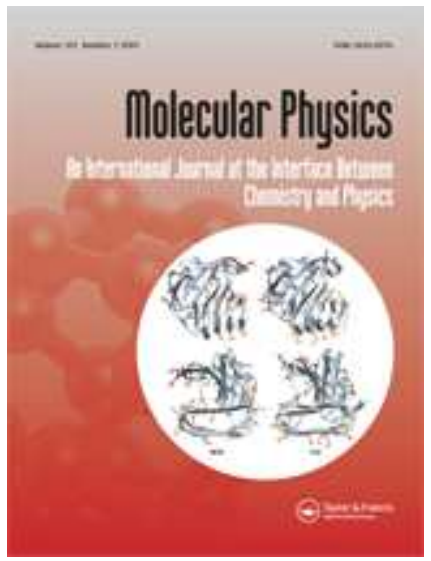

\section{Strong-field control of electron localization during molecular dissociation}

\begin{tabular}{|c|c|}
\hline Journal: & Molecular Physics \\
\hline Manuscript ID: & TMPH-2007-0329.R1 \\
\hline Manuscript Type: & Invited Article \\
\hline $\begin{array}{r}\text { Date Submitted by the } \\
\text { Author: }\end{array}$ & 06-Dec-2007 \\
\hline Complete List of Authors: & $\begin{array}{l}\text { Kling, Matthias; FOM Instituut voor Atoom en Molecuul Fysica } \\
\text { (AMOLF); Max-Planck Institut für Quantenoptik } \\
\text { Siedschlag, Christian; FOM Instituut voor Atoom en Molecuul Fysica } \\
\text { (AMOLF) } \\
\text { Znakovskaya, Irina; Max-Planck Institut für Quantenoptik } \\
\text { Verhoef, Aart; Max-Planck Institut für Quantenoptik } \\
\text { Zherebtsov, Sergey; Max-Planck Institut für Quantenoptik } \\
\text { Krausz, Ferenc; Max-Planck Institut für Quantenoptik; Ludwig- } \\
\text { Maximilians-Universität München, Department für Physik } \\
\text { Lezius, Matthias; Max-Planck Institut für Quantenoptik } \\
\text { Vrakking, Marc; FOM Instituut voor Atoom en Molecuul Fysica } \\
\text { (AMOLF) }\end{array}$ \\
\hline Keywords: & Strong-field control, Few-cycle laser pulses, Charge localization \\
\hline \multicolumn{2}{|c|}{$\begin{array}{l}\text { Note: The following files were submitted by the author for peer review, but cannot be converted } \\
\text { to PDF. You must view these files (e.g. movies) online. }\end{array}$} \\
\hline \multicolumn{2}{|l|}{$\begin{array}{l}\text { figures.zip } \\
\text { MolPhys_2007_Figures_5.ppt }\end{array}$} \\
\hline
\end{tabular}




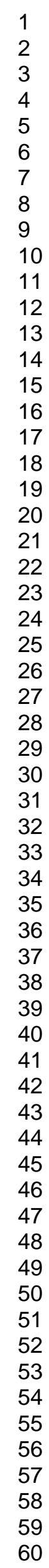

\section{$\$$ Manuscript Central}

URL: http://mc.manuscriptcentral.com/tandf/tmph 
Catchline (head of first page only) Molecular Physics, Vol. X, No. X, Month 2008, xxx-xxx

Running heads (verso) M.F. Kling et al.

(recto) Strong-field control of electron localization during molecular

dissociation

Article Type (e.g. Research Article)

\title{
Strong-field control of electron localization during molecular dissociation
}

\author{
M.F. Kling ${ }^{1,2^{*}}$, Ch. Siedschlag ${ }^{1}$, I. Znakovskaya, ${ }^{2}$ A.J. Verhoef, ${ }^{2}$ S. Zherebtsov, ${ }^{2}$ F. Krausz, ${ }^{2,3}$ M. Lezius, ${ }^{2}$ \\ M.J.J. Vrakking ${ }^{1 *}$ \\ ${ }^{1}$ FOM Instituut voor Atoom en Molecuul Fysica (AMOLF), Kruislaan 407, 1098 SJ Amsterdam, Netherlands \\ ${ }^{2}$ Max-Planck-Institut für Quantenoptik, Hans-Kopfermann-Strasse 1, D-85748 Garching, Germany \\ ${ }^{3}$ Department für Physik, Ludwig-Maximilians-Universität München, Am Coulombwall 1, D-85748 Garching, \\ Germany \\ *E-mails: matthias.kling@mpq.mpg.de, m.vrakking@amolf.nl \\ ${ }^{ \pm}$Dedicated to Prof. Raphy Levine on the occasion of his 70th anniversary.
}

\begin{abstract}
We demonstrate how the waveform of light can be used to control a molecular dissociation by steering and localization of electrons. Experimental results have been obtained for the dissociative ionization of the homonuclear and heteronuclear Hydrogen derivates $\mathrm{D}_{2}$ and HD. Asymmetric ejection of the ionic fragments reveals that light-driven electronic motion prior to dissociation localizes the electron on one of the two ions in the diatomic molecular ions in a controlled way. Extension of these results to electron transfer in complex molecules suggests a new paradigm for controlling photochemistry.
\end{abstract}

Keywords: Strong-field control, few cycle laser pulses, charge localization AMS Subject Classification: 81V55; 81 V80 


\section{Introduction}

Coherent control of molecular dynamics has entered a new and exciting regime with the advent of intense few-cycle phase stabilized laser pulses [1]. Laser technology now allows for the generation and control of electromagnetic fields, where the electric field can be switched between 0 and several a.u. with a temporal accuracy of a few 100 as [2]. Obviously, only electrons can respond on this timescale, and atomic centers will remain frozen. If the laser intensity is chosen carefully, the extreme non-linearity of the strong field tunneling probability due to the Gamov-factor $\exp \left(-2\left(2 U_{\mathrm{I}}\right)^{3 / 2} / 3|E(t)|\right)$, with $U_{\mathrm{I}}$ the ionization potential and $E(t)$ the electric field, leads to situations where electrons are liberated from a molecule within a fraction (100-300 as) of the cycle of the carrier wave. A full cycle of this carrier wave typically lasts about 2660 as at $800 \mathrm{~nm}$ when using Ti:Sapphire lasers. Subsequently, these electrons are driven by the laser field [3], which leads to daughter processes that can, in principle, be precisely synchronized with respect to the original ionization event. Typical cases are recombination and high-order harmonic generation [4], scattering and high energy above-threshold ionization (ATI) [5, 6], as well as attosecond electron diffraction [7]. All of these processes take place about $1 / 3$ of the laser cycle after strong field ionization (SFI) has happened close to the peak electric field [3], when the electron revisits its parent near a zero-crossing of the electric field. The electron rescattering process can also lead to population transfer into excited states above the ionic ground state, which is usually prepared during the strong field tunneling process. This is especially attractive to molecular physics, because the preparation of higher excited molecular states can thus be very precisely timed. Furthermore, because electron rescattering is approximately equal to classical electron impact excitation of ions, no strong selection rules apply, in contrast with the optical case. Only if during rescattering recombination takes place, will the molecule preferentially end up in its initial state, and excess energy will be given away as harmonic radiation. This has been used with advantage for the prominent tomographic imaging of molecular orbitals by Itatani et al. [8].

Molecular electron rescattering physics can and has been investigated intensively by various groups in recent years with multi-cycle laser pulses. However, for the case that the laser pulse duration approaches the optical period one enters the few-cycle regime and the electromagnetic driver fields become increasingly asymmetric. Such fields have recently opened up new avenues for coherent control. Spatial control of electron emission has been observed and has become a major tool for long term stabilization of the laser phase [9]. It has also been possible to control total fragment particle momenta [10]. The prerequisite for such experiments, control of the carrier-envelope phase (CEP) itself has become available as a laser control parameter since the groundbreaking work of T. Hänsch and coworkers [11], and its extension towards amplified laser systems by A. Baltuska et al. [2]. The latter has paved the way into the strong field community. Stabilization and control of the laser phase with comparably high precision has made many experiments possible that are directly related to attosecond physics [12-18]. The relation between the CEP and attosecond physics itself can be easily understood, since control over the CEP is virtually equal to control of a light field with attosecond precision. CEP control applied to the few-cycle regime, however, enables access to mono-cycle strong field ionization. In such cases, subsequent steering of isolated attosecond electron wavepackets is feasible and gives access to controlled time dependent and intense polarization of the target system. In combination with molecular alignment or orientation selection via SFI the technique can be used to control the final localization of charge during the molecular dissociation, as has been previously demonstrated for the D-D homonuclear dimer [15]. In this paper, we extend the discussion of such experiments towards the heteronuclear dimer H-D, and towards additional aspects in the CEP control of charge redistribution, which may be attributed to phase control of bond-softening processes. 
Hydrogen ionization and dissociation has been attractive to the femtosecond community for several years (see e.g. [19] and references therein). Some reasons for this are: First, $\mathrm{H}_{2}$ intranuclear vibrational wavepacket dynamics is very fast and requires a temporal resolution in the few-fs regime [20]. Second, because only two electrons and two protons are involved, the system can be numerically accessed with high accuracy [21]. As such, it has model character for the treatment of more complex molecules and with regard to chemistry perhaps more interesting cases. Third, lower ionic levels in Hydrogen are energetically well separated [22, 23]. Because of this, IR multiphoton ionization ends up mostly in one single electronic state $\left(1 \mathrm{~s}_{\mathrm{g}}{ }^{+}\right)$. Subsequent electron rescattering events then populate a superposition of higher states, e.g. create a synchronized electronic wavepacket. The corresponding coupled electron-nuclear dynamics evolving within the rapidly decaying strong laser field can be made responsible for final charge localization [15, 24-26].

\section{Experimental}

The experimental scheme that was used here has been described earlier [15]. In brief, transform-limited laser pulses of 25 fs duration with $1 \mathrm{~mJ}$ pulse energy have been generated with a $3 \mathrm{kHz}$ phase stabilized amplified Ti:sapphire laser system (Femtolasers, Femtopower Compact Pro). The pulse was spectrally broadened using a one-meter long hollow-core fiber of $250 \mu \mathrm{m}$ diameter filled with $3.8 \mathrm{bar}$ Neon gas. The laser pointing into the fiber was controlled with high precision with a home-built stabilizer system consisting of a CCD camera and a motorized mirror mount. The output pulses from the fiber exhibited a significantly broadened spectrum (see e.g. [27]) and were compressed down to a near-transform limited duration of $\sim 5$ fs using 8 reflections in a chirped mirror compressor. By tuning of the gas pressure in the hollow-core fiber the pulse length was adjusted precisely between 25 and 5 fs. The pulse duration was monitored online with a commercial dispersion balanced autocorrelator. The laser phase has been stabilized with a feedback loop described in [28, 29]. The polarization is rectified using three $5 \mu \mathrm{m}$ thick pellicles at Brewster angle. Fine tuning of the pulse duration and varying the CEP was done by changing the amount of material dispersion with a pair of fused silica wedges after the hollow fibre. The phase jitter was smaller than $150 \mathrm{mrad}$. Phase-stabilized, linearly polarized pulses at a central wavelength of $760 \mathrm{~nm}$ were focused with a spherical mirror $(\mathrm{R}=80 \mathrm{~cm})$ into the center of the ion optics of a velocity-map imaging spectrometer [30]. In the focus intensities of up to $5 \cdot 10^{14} \mathrm{~W}$ $\mathrm{cm}^{-2}$ have been realized, and an adjustable iris has been used to vary the intensity in the focus. Ions and electrons were generated at the crossing point between the laser and the molecular beam (with a particle density of appr. $10^{13} \mathrm{~cm}^{-3}$ ) and were accelerated and focused with the ion optics onto a MCP-phosphor screen assembly (Hamamatsu, F2226-24PX). The molecular beam was produced by a pulsed nozzle of $1 \mathrm{~mm}$ diameter and operated at $50 \mathrm{~Hz}$. The molecular beam was differentially pumped and passed a skimmer of 1 $\mathrm{mm}$ diameter $10 \mathrm{~cm}$ downstream before entering the interaction region. The sensitivity of the MCP-detector was switched so that only ions or electrons from every 60th laser pulse were detected. This allowed for a low background pressure in the chamber (typically $2 \cdot 10^{-7} \mathrm{mbar}$ ). The velocity map images have been recorded with a CCD camera (Pulnix, TM-9701) and were typically averaged over $60 \mathrm{~s}$ of data acquisition for each setting of the laser phase.

Figure $1 \mathrm{~b}$ shows a typical experimental momentum map of $\mathrm{D}^{+}$ions recorded with $5 \mathrm{fs}$ excitation at $10^{14} \mathrm{~W}$ $\mathrm{cm}^{-2}$ without CEP stabilization. The laser was polarized along the $p_{\mathrm{y}}$-axis. Two main contributions are visible in both the upwards and the downwards emission direction: a strong peak at lower energies with a relatively narrow angular distribution and a contribution at larger momenta with a wide angular distribution. After an inversion of the image using an iterative inversion procedure [31], the original 3D-momentum distribution can be reconstructed. By integration of the inverted image over the full solid angle the energy spectrum displayed in Figure $2 \mathrm{~A}$ is derived. The spectral features can be attributed to the following reaction pathways (as sketched in Figure 2C): 
A) Following strong-field tunnel ionization of $\mathrm{D}_{2}$ with the production of an electron and a $\mathrm{D}_{2}{ }^{+}$ion in the $1 \mathrm{~s} \sigma_{\mathrm{g}}{ }^{+}$-state, recollisional excitation (RCE) $[32,33]$ to the $2 \mathrm{p}_{\mathrm{u}}{ }^{+}$state by the returning electron leads to dissociation and formation of $\mathrm{D}^{+}$and $\mathrm{D}$ fragments with energies above $3 \mathrm{eV}$. This channel shows a broad angular distribution as seen in Figure 2B in agreement with earlier observations.[34] Note that no ions at these energies are observed with circular polarized light (Fig. 2A), strongly supporting recollision to be responsible for their production.

B) Close to the outer turning point of the nuclear wavepacket bond softening (BS) $[19,35,36]$ becomes a prominent process, leading to very low fragment energies below $3 \mathrm{eV}$ with its main contribution between 0 and $2 \mathrm{eV}$.

C) At the intensities used in these studies, Coulomb explosion of $\mathrm{D}_{2}$ with the production of two $\mathrm{D}^{+}$ions is possible via enhanced ionization (EI). This channel is, however, unwanted for the present investigations and therefore we tried to keep such signals at a minimum. In fact, only a minor contribution of EI is seen between 2 and $3 \mathrm{eV}$, which exhibits a smaller angular distribution than the BS pathway (see Figure 2B).

\section{$\underline{\text { Results I: electron localization in } \mathrm{D}_{2}}$}

In order to elucidate the role of the CEP on the experimental ion momentum distributions, the laser phase was scanned carefully over a range of multiple cycles. The angle-integrated asymmetry in the ion momentum distribution at a certain energy $\mathrm{W}=\mathrm{p}^{2} /(2 \mathrm{~m})$ and phase $\varphi$ was obtained from

$A(W, \varphi)=\frac{P_{u p}(W, \varphi)-P_{\text {down }}(W, \varphi)}{P_{u p}(W, \varphi)+P_{\text {down }}(W, \varphi)}$

with

$P_{\text {up }}(W, \varphi)=\int_{330}^{360} \int_{0}^{360} P(W, \theta, \phi, \varphi) \sin \theta d \theta d \phi+\int_{0}^{30360} \int_{0}^{36} P(W, \theta, \phi, \varphi) \sin \theta d \theta d \phi$

and

$P_{\text {down }}(W, \varphi)=\int_{150}^{210} \int_{0}^{360} P(W, \theta, \phi, \varphi) \sin \theta d \theta d \phi$,

with $\theta$ and $\varphi$ being the polar and azimuthal angles, respectively. We chose to analyze the ion emission within a restricted angular range because our ability to control electron motion in hydrogen requires that the laser couples the two lowest-lying electronic states. For molecules aligned orthogonally to the laser polarization axis, this coupling would be absent.

The contour plot in Figure 3A shows the measured angle-integrated asymmetry $A\left(W, \varphi_{\mathrm{CEP}}\right)$ for dissociative ionization of $\mathrm{D}_{2}$ into $\mathrm{D}^{+}+\mathrm{D}$ as a function of the carrier-envelope phase $\varphi_{\text {CEP }}(\mathrm{x}$-axis) and the kinetic energy $W$ of the $\mathrm{D}^{+}$ion fragment (y-axis, see also Figure 2). Note that here the laser phase is only given as a relative number as the absolute phase has not been determined. Figure 3A shows that in an energy range between 3 and $8 \mathrm{eV}$ locking of the laser phase in the few-cycle limit causes a remarkable asymmetry in the upward and downward emission. Regions in Figure 3A where the asymmetry oscillates as a function of the phase represent final energies of the $\mathrm{D}^{+}$ions where the direction of their emission is effectively controlled by the sub-cycle evolution of the laser field driving the photodissociation. The extent of this ability to control is further illustrated in Fig. 3B, which displays asymmetries that are integrated over selected energy intervals. 
The highest degree of asymmetry, with a modulation depth of up to $45 \%$, is observed between 3 and $8 \mathrm{eV}$. Above $8 \mathrm{eV}$, the asymmetry appears to cease completely. A very small phase dependence is seen between 1 and $2 \mathrm{eV}$ (see also Figure 3B), which represents the typical energy range for bond softening. Most interestingly, this low energy channel for charge localization appears to be out of phase by ca. $\pi / 2$ with respect to the high energy channel. In principle, when using 5 fs laser pulses, bond softening would not be expected, since this process requires motion of the vibrational wave packet that is formed by the tunnel ionization to the outer turning point of the $1 \mathrm{~s}_{\mathrm{g}}{ }^{+}$potential well. This is expected to take about half a vibrational period, i.e. $12 \mathrm{fs}$ in the case of $\mathrm{D}_{2}$ [37]. However, in our experiments the pulse contrast was not ideal. A background present in the few-cycle pulses amounted to ca. $10 \%$ in intensity at times when the internuclear distance moves into the BS region and might contribute to the asymmetry [26].

Plots of the expectation value of the alignment parameter $\left\langle\cos ^{2}(\theta)>\right.$ versus the phase $\varphi_{\text {CEP }}$ are given in Fig. $3 \mathrm{C}$ and show the degree of alignment of $\mathrm{D}^{+}$ions within the energy ranges used in Fig. 3B. A strong phase dependence is again seen for the energy range between 3 and $8 \mathrm{eV}$. This phase dependence is not present in the low energy contribution. A comparison of Figs. 3B and 3C indicates that the observation of a large asymmetry between 3 and $8 \mathrm{eV}$ correlates with a higher degree of alignment. The relation between the charge localization process and the angular distribution of the fragment ions is further explored in Figure 4A, where the amplitude $A_{0}(W, \theta)$ of the asymmetry oscillation $A\left(W, \theta, \varphi_{\text {CEP }}\right)=A_{0}(W, \theta) \sin \left(\varphi_{\text {CEP }}+\varphi_{0}(W, \theta)\right)$ is shown as a function of kinetic energy $\mathrm{W}$ and fragment angle $\theta$. Clearly, for fragment angles $\theta>50$ degrees the asymmetry vanishes. Moreover, a butterfly shape of the asymmetry amplitude is apparent and indicates different mechanisms for the generation of the asymmetry at low and high energies. This will be discussed in more detail below. Additionally, Fig. 4B shows the asymmetry dependence on the pulse duration, which declines quasi-exponentially from ca. $45 \%$ close to 5 fs towards $1 \%$ above $9 \mathrm{fs}$. This behavior reveals that the asymmetry of the field is driving the charge localization process and that few-cycle pulses are an important prerequisite for the electron localization control that has been achieved.

\section{Theoretical interpretation of charge localization effect in $\mathrm{D}_{2}$}

The electron localization control in the fragmentation of $\mathrm{D}_{2}$ arises due to a phase control mechanism that consists of two parts. The first part has already been partially discussed in relation to the results shown in Figure 2A. The absence of fragments in the kinetic energy range between 3 and $8 \mathrm{eV}$ (where the observed asymmetry is most pronounced) in experiments using circularly polarized light strongly suggests the involvement of a re-collision of the electron that is ejected in the tunnel ionization that produces the $\mathrm{D}_{2}{ }^{+}$ion. Our interpretation is that re-collision of this electron with the $\mathrm{D}_{2}{ }^{+}$ion leads to excitation of the $\mathrm{D}_{2}{ }^{+}$ion from the $1 \mathrm{~s} \sigma_{\mathrm{g}}{ }^{+}$-state to the dissociative $2 \mathrm{p \sigma}_{\mathrm{u}}^{+}$state. Further, indirect support for this is provided by the observation of $\mathrm{D}^{+}$fragments with a kinetic energy of up to $12 \mathrm{eV}$, consistent with acceleration along a repulsive curve starting from an internuclear distance close or equal to the internuclear distance in the neutral ground state. However, this re-collision excitation by itself is not enough to cause an asymmetry. If the observation of a high kinetic energy $\mathrm{D}^{+}$fragment could be used as an indication of the fact that a fragment had been detected that was dissociating along the $2 \mathrm{p \sigma}_{\mathrm{u}}^{+}$potential curve, then the molecule would retain its parity up to the point of detection, and - parity being a symmetry property of the electronic wave function - the distribution of the electron over the two $\mathrm{D}^{+}$ions involved in the dissociation would necessarily have to be symmetric. A second ingredient is required, which breaks the parity of the electronic wave function. As proposed in ref. [15], and subsequently confirmed in more detailed theoretical treatments [21,25] laser-induced coupling between the $2 \mathrm{p \sigma}_{\mathrm{u}}{ }^{+}$state and the $1 \mathrm{~s} \sigma_{\mathrm{g}}{ }^{+}$-state can convert the dissociative wave packet that starts out on the $2 \mathrm{p \sigma}_{\mathrm{u}}^{+}$state into a 
coherent superposition state containing contributions from both the $2 \mathrm{p \sigma}_{\mathrm{u}}{ }^{+}$state and the $1 \mathrm{~s} \sigma_{\mathrm{g}}{ }^{+}$-state and importantly - with a broken parity.

According to the simplified semiclassical model presented in [15] the time evolution of the wave function for the Hydrogen molecule after recollision can be calculated by expanding the full wave function for the electronic coordinate and the internuclear distance in terms of the lowest lying electronic states,

$$
\Psi(\vec{r}, R ; t) \approx|g\rangle \psi_{g}(R ; t)+|u\rangle \psi_{u}(R ; t),
$$

where $|g\rangle$ and $\mid u>$ correspond to the $1 s \sigma_{g^{+}}$and the $2 p \sigma_{u^{+}}$states, respectively, and where $\psi_{g / u}$ represent the corresponding nuclear wave packets. In accordance with our observation that the asymmetry is primarily detected for fragments that are ejected along the laser polarization axis, the molecule is assumed to be aligned along the axis of the laser field. Further support for this assumption comes from the fact that aligned molecules are preferentially ionized via SFI. By inserting this Ansatz into the time-dependent Schrödinger equation one obtains the coupled equations

$$
i \frac{\partial}{\partial t}\left(\begin{array}{l}
\psi_{g}(R ; t) \\
\psi_{u}(R ; t)
\end{array}\right)=\left(\begin{array}{cc}
\frac{-1}{M} \frac{\partial^{2}}{\partial R^{2}}+V_{g}(R) & V_{g u}(R) \\
V_{g u}^{*}(R) & \frac{-1}{M} \frac{\partial^{2}}{\partial R^{2}}+V_{u}(R)
\end{array}\right)\left(\begin{array}{l}
\psi_{g}(R ; t) \\
\psi_{u}(R ; t)
\end{array}\right)
$$

with the binding potential curve $V_{g}(R)$, the dissociative curve $V_{u}(R)$ and the coupling between them $V_{g u}(R)$. Tabulated values for the potential curves were used [22]. Integration of eq. 5 yields the time-dependent nuclear wave functions. The initial condition directly after the recollision consists of placing the vibrational ground state, obtained by relaxation on the respective potential curve [23], onto the dissociative potential curve of the molecular ion $\left(2 p \sigma_{u+}\right)$. The recollision time for the first recollision is $1.7 \mathrm{fs}$ after ionization [32]. Due to the fact that the experiment employed few-cycle pulses, later recollision events are considered to be efficiently suppressed [21,34]. We note that the ionization is considered as a single event that occurs at the maximum of the laser electric field. This is a simplification, since the application of ADK theory [38] would predict that the ionization may occur during more than a single half-cycle of the laser, and furthermore, during a finite time interval within each half-cycle. A very rigorous theoretical treatment of the dynamics that goes well beyond the approach here and includes the ionization and recollision steps was recently presented by Gräfe and Ivanov [25]. However, a computational treatment of the hydrogen dissociation starting with an ADK treatment of the ionization is beyond the scope of the present paper, where our main aim is to qualitatively explain the physics responsible for the observed phase-control.

In the approach used here, for the calculation of the asymmetry, the electronic basis is changed to two states that are localized on the left and on the right nucleus, respectively. Without loss of generality, we define

$$
\begin{aligned}
& |l\rangle=\frac{1}{\sqrt{2}}(|g\rangle+|u\rangle) \\
& \text { and } \\
& |r\rangle=\frac{1}{\sqrt{2}}(|g\rangle-|u\rangle) .
\end{aligned}
$$

By projecting onto these states, the corresponding nuclear wave functions are obtained. 
From these expressions the (t- as well as R-dependent) the probabilities for electron to remain on the left or on the right atom are calculated

$$
\begin{aligned}
& P_{l}(R, t)=\frac{1}{2}\left|\psi_{g}(R ; t)+\psi_{u}(R ; t)\right|^{2}, \\
& P_{r}(R, t)=\frac{1}{2}\left|\psi_{g}(R ; t)-\psi_{u}(R ; t)\right|^{2} .
\end{aligned}
$$

The time-dependent electron localization parameter is then defined as

$\int\left(P_{l}(R, t)-P_{r}(R, t)\right) d R$ $\int\left(P_{l}(R, t)+P_{r}(R, t)\right) d R$.

From these expressions we can immediately see that it is the coherent superposition of the two electronic states $1 s \sigma_{g+}$ and $2 p \sigma_{u^{+}}$that is responsible for the asymmetry in the charge localization.

In Figure 5, the temporal evolution of the laser field and the time-dependent electron localization parameter quantifying the localization on the upper/lower nucleus is displayed. The initial asymmetry that develops in the electron density is synchronized to the laser frequency, the intuitive picture being that the laser drives the electron back-and-forth (on attosecond timescales) between the two nuclei. However, as the molecule dissociates, the oscillatory motion of the electron between the two nuclei is impeded by the emergence of a potential barrier between the two nuclei. For an internuclear distance which is close to the internuclear distance where enhanced ionization would occur the electron oscillations stops and the electron density is found to localize predominantly on one of the atoms. In agreement with the experimental observation shifting the CEP by $\pi$ turns the laser field and thus the asymmetry around.

The observation of asymmetric $\mathrm{D}^{+}$emission as a result of electron localization requires that in our velocityresolved $\mathrm{D}^{+}$measurements we are unable to identify the quantum path (i.e. the $1 s \sigma_{g+}$ or the $2 p \sigma_{u+}$ curve) along which the measured ions were created. This restricts the kinetic energy range where an asymmetric emission may be expected. In good agreement with the experiment, the asymmetry is calculated to peak at around $6 \mathrm{eV}$. Importantly, no electron localization is observed at the very highest kinetic energies that occur in the experiment, since the wave packet that dissociates on the $1 s \sigma_{g+}$ curve is necessarily slower than the wave packet that dissociates on the repulsive $2 p \sigma_{u^{+}}$curve. In the intermediate energy range between 2 and $8 \mathrm{eV}$ the charge localization phase dependence exhibits, if at all, only weak substructures. This behavior can be easily understood from the electron rescattering process, which allows for access to a broad energetic range in molecular excitation during the sub-cycle rescattering event. The interpretation of the low KER regime is, however, more complicated. Simulations in [21] show a larger phase offset of $\sim \pi$ between BS and RCE. As 
suggested by Roudnev and Esry [39], asymmetries could be the result of pure interference between the $1 s \sigma_{g^{+}}$ and $2 p \sigma_{u^{+}}$molecular channels populated directly via sequential optical excitation. If the nuclei are dissociating in two different molecular channels, they can still contribute at the same kinetic energy. In the few cycle case, laser bandwidth and Stark-shifting of the initial state may be strong enough to create this overlap of nuclear wavepackets, so that the relative phase between the final components still depends on the CEP.

Another quite interesting aspect comes into play from the $\theta$-dependence of the asymmetry as illustrated in Figures $2 \mathrm{~B}$ and $4 \mathrm{~A}$. Within our simplified model, interference at low dissociation energies means that the low energy tail of the dissociative excited state wavepacket is interfering with a wavepacket that must have been stimulated from $2 p \sigma_{u^{+}}$to $1 s \sigma_{g+}$ by the laser field relatively early after the rescattering excitation process. We suggest that early de-excitation should be more dependent on the molecular alignment, which would explain that in Figure 4 the low energy region populates a smaller angular range. On the other hand, interference at high energy means that the downward transition takes place relatively late. When the gap between the two potential curves becomes less than a photon, it should suddenly become very difficult to induce a transition and only the very well aligned molecules may still succeed. Again, the angular selection becomes stricter, as observed in our experiment.

To conclude, within our modeling, we understand final charge localization during molecular dissociation in the following way. The molecular ions are formed in a (single) ionization event that occurs at the maximum of the laser electric field. The ionization event starts a vibrational wave packet in the $1 s \sigma_{g+}$ ground electronic state of $\mathrm{D}_{2}^{+}$that mimics the vibrational ground state wave function of $\mathrm{D}_{2}$ before excitation. Rescattering then leads to population transfer from the $1 s \sigma_{g+}$ ground electronic state to the $2 p \sigma_{u+}$ excited electronic state at a delay of $\sim 1.7 \mathrm{fs}$ [32] after ionization. Because of the strongly repulsive nature of the $2 p \sigma_{u^{+}}$state, the excited $\mathrm{D}_{2}{ }^{+}$molecule rapidly dissociates and the resulting fragments acquire significant kinetic energies up to $10 \mathrm{eV}$. During the molecular dissociation the laser field can, however, transfer part of the $2 p \sigma_{u^{+}}$population back into the $1 s \sigma_{g+}$ state, thereby producing a dissociative wave packet with large excess kinetic energy. The emerging coherent superposition of the two electronic states results in a time-dependent localization of the electron density on the upper or lower nucleus due to the gerade and ungerade nature of the two states.

\section{Results II: electron localization in HD}

Following the experimental demonstration of CEP control of electron localization in $\mathrm{D}_{2}$ further experiments were performed exploring the possibility to control electron localization in HD. Figure 6A shows the $\mathrm{D}^{+}$ion kinetic energy spectrum that is obtained after excitation of $\mathrm{HD}$ with $5 \mathrm{fs}$ laser pulses at $10^{14} \mathrm{~W} \mathrm{~cm}^{-2}$. Unlike the case of $\mathrm{D}_{2}$ the HD measurements were successfully accompanied by a measurement of above-threshold ionisation in Xe, allowing to assign a CEP of $\pi / 5$. The insert depicts a typical velocity map image, from which the energy spectrum has been obtained after angular integration. By varying the CEP an asymmetry map $A\left(W, \varphi_{\mathrm{CEP}}\right)$ was obtained (see Fig. $\left.6 \mathrm{~B}\right)$. In comparison to the homonuclear $\mathrm{D}_{2}$ case, quite similar asymmetries are obtained for the heteronuclear molecule. Figure 7A shows related data for $\mathrm{H}^{+}$from $\mathrm{HD}$ for the same excitation conditions $\left(5 \mathrm{fs}, 10^{14} \mathrm{~W} \mathrm{~cm}^{-2}\right)$. Note that the energy spectra differ by approximately a factor of $\sqrt{2}$ due to momentum conservation during the dissociation process. In general, proton spectra tend to show more noise, because of an increased background from ionization of $\mathrm{H}_{2} \mathrm{O}$. Apart from this additional noise, the $\mathrm{D}^{+}$and $\mathrm{H}^{+}$ion spectra shown in Figures 6 and 7 show very comparable asymmetry features. The asymmetries are also very similar to the homonuclear case displayed in Figure 3. As seen in Figure 6B, for $\mathrm{D}^{+}$the asymmetry becomes prominent from 2 to $6 \mathrm{eV}$ and for $\mathrm{H}^{+}$shown in Figure 7B between 3 and $8 \mathrm{eV}$. Note that the phase features of $\mathrm{H}_{\Delta}^{+}$and $\mathrm{D}_{\Delta}^{+}$in figures 6 and 7 do not coincide in full, which is possibly due to the low 
signal-to-noise ratio in the $\mathrm{H}^{+}$measurements. As for $\mathrm{D}_{2}$, an asymmetry oscillation is also observed for $\mathrm{HD}$ at lower ion kinetic energies within the range of the bond softening contribution (0.7-1.5 and 1-2 eV for $\mathrm{D}^{+}$and $\mathrm{H}^{+}$, respectively). Again, a shift of the phase of the asymmetry oscillation between the low and high energy channels of approximately $\pi / 2$ is seen (see figures $6 \mathrm{C}$ and $7 \mathrm{C}$ ).

Figure 8 shows the energy and angular dependence of the amplitude $A_{0}(W, \theta)$ of the asymmetry oscillation $A\left(W, \theta, \varphi_{\mathrm{CEP}}\right)=A_{0}(W, \theta) \sin \left(\varphi_{\mathrm{CEP}}+\varphi_{0}(W, \theta)\right)$ that was obtained for $\mathrm{D}^{+}$ions from the dissociative ionization of HD. Similar to Figure $4 \mathrm{~B}$, where this analysis was performed for $\mathrm{D}_{2}$, the asymmetry is restricted to angles $\theta<50$ degree and shows a significant difference in the angular distribution of the asymmetry between the low $(0.7-1.5 \mathrm{eV})$ and high energy (above $2 \mathrm{eV}$ ) channels. The kinetic energy range where the asymmetries are observed for $\mathrm{H}^{+}$and $\mathrm{D}^{+}$from $\mathrm{HD}$ is lower than the kinetic range where these effects were observed for $\mathrm{D}_{2}$. A possible reason for this may be the fact that the vibrational period of $\mathrm{HD}$ is shorter than that of $\mathrm{D}_{2}$, meaning that the vibrational wave packet that is initially produced in the $1 s \sigma_{g}$ ground electronic state moves farther out during the $1.7 \mathrm{fsec}$ separating the ionisation and the recollision event. If so, the recollision excitation promotes the nuclear wave packet to a somewhat lower position on the repulsive $2 p \sigma_{l_{l}}$ curve.

The CEP in Figures 6 and 7 has been determined in situ as an absolute phase via a reference measurement of the asymmetry $A\left(W, \varphi_{\mathrm{CEP}}\right)$ in the electron emission in above-threshold ionization (ATI) of Xe (see Figure $9 \mathrm{~A}$ for the ATI spectrum and 9B for the asymmetry map). The CEP was set to zero at positions where the cut-off electron emission reaches its maximum in the upward direction [40]. This should facilitate direct comparison to theoretical studies of the system. Interestingly, in HD, in agreement with recent theoretical findings [21], the emission of ions to one of the two sides of the laser polarization does not necessarily coincide with phase values of $\varphi_{\text {CEP }}=n \cdot \pi($ with integer number $n)$.

The phase difference between the high-energy (recollision) and low-energy (bond softening) channels in both $\mathrm{D}_{2}$ and HD of $\sim \pi / 2$ is reminiscent to shifts that have been reported between direct and rescattered ATI photoelectron spectra for rare gas atoms, which for the direct (low energy) electrons have been explained as manifestation of double slits in time [14]. In the present experiment, however, the mechanism is slightly different. Based on inspection of the kinetic energy distributions in Figures 6 and 7 we have attributed the asymmetries at low energies to the onset of contributions from a (direct) bond softening (BS) channel, while higher energies have been attributed to (indirect) recollisional excitation (RCE). The difference in the ionization mechanism is therefore in principle similar to [14]. However, in the molecular case the closely coupled electron-nuclear dynamics has to be additionally taken into account. Thus, the asymmetry of the BS channel should rather be understood in terms of an n-photon pathway interference between the two respective trajectories for the dissociation of the Hydrogen molecular ion. During evolution of the molecule the laser field can couple the gerade and the ungerade states directly when the wavepacket approaches the outer potential well [26]. This coupling can be made responsible for the observed phase dependence as calculated by Roudnev and Esry for $\mathrm{HD}^{+}$[39]. Moreover, weak phase shifted asymmetries for total ion kinetic energies below $5 \mathrm{eV}$ have been observed by [21] in $\mathrm{D}_{2}$ model calculations, and have been attributed to asymmetric dissociation as described in [24].

\section{Conclusion}

Electron transfer processes play a pivotal role in chemistry. Presently, following the generation and measurement of single sub-femtosecond pulse made possible by unprecedented control of bound and free atomic electrons, respectively, with the sub-cycle evolution of a strong light field [41], it may be become
Gelöscht:

Gelöscht:

Gelöscht: In this respect it should be mentioned that similar n-photon pathway interferences have been suggested by Roudnev and Esry [39].

Formatiert: Hochgestellt 
possible to observe electron transfer processes on the fastest timescales that these processes take place. When attosecond pulses are used to initiate electron dynamics in molecules, the high photon energy of the attosecond pulse generally results in ionization. As discussed by Remacle and Levine [42], removal of an electron on attosecond timescales will often result in the formation of electronic wave packets, because the electron-hole density that results from removing an electron from the highest-occupied molecular orbital (HOMO) does not match the electron-hole density in the singly occupied HOMO of the cation formed on ionisation. Ultrafast removal of an electron therefore not only forms the ground electronic state of the cation, but, rather, a coherent superposition of electronic states. Remacle and Levine [42] have argued that the formation of this wave packet may lead to electron transport across the ionic structure that is formed. For example, the photoionization of the neutral tetrapeptide molecule TrpLeu 3 is expected to lead to the population of the HOMO-1 and the HOMO of the $\mathrm{TrpLeu}_{3}{ }^{+}$cation. The shape of these orbitals and the 3-eV energy splitting between the two orbitals suggest that electron transfer from one end of the molecule to the other occurs in less than $1 \mathrm{fs}$. Comparing a wide range of electronic systems, Breidbach and Cederbaum [43] observed that the sudden removal of an electron is accompanied by a characteristic time response completed in approximately 50 as. This time response is interpreted in terms of a filling - upon ionization - of the exchange-correlation hole associated with the electron ionized by its neighbouring electrons.

The time that sub-femtosecond pulses are used to initiate the formation and subsequently probe the formation of an electron wavepacket that transfers electron density across a large molecule has not yet come. However, in the present paper we have extended sub-femtosecond electron control to molecules and obtained first evidence of its usefulness in controlling reaction dynamics. We have controlled the dissociation of $\mathrm{D}_{2}^{+}$and $\mathrm{HD}^{+}$by steering electron wave packet motion with the sub-cycle, i.e. sub-femtosecond evolution of the electric field of a few-cycle light wave. A coherent superposition of two electronic states in the molecular ion is responsible for an oscillating electron density and the final localization of the electron. While the computed electron and nuclear dynamics are consistent with our measurement, deeper insight into the role of fieldcontrolled electron dynamics in driving chemical reactions will require time-resolved investigations $[25,44$, 45]. The door to such studies is now open thanks to the availability of sub-femtosecond extreme-ultraviolet (XUV) pulses synchronized with the few-cycle control pulse [41]. Synthesized ultrawide-band (multi-color) waveforms (comprising near-infrared, visible and possibly ultraviolet light), which can now both be produced and measured may dramatically enhance the efficiency of steering reactions by creating electronic wave packets and subsequently driving them towards selected sites in complex molecular systems. Indeed, recent theoretical work suggests that circular electronic motion in ring-shaped molecules can be induced by controlled light-fields [46].

Electron transfer processes are extremely important in chemistry and biology. For example, rapid electron transfer can promote both damage and repair of DNA base-pairs. Our results for the intense-field dissociative ionization of $\mathrm{D}_{2}$ and $\mathrm{HD}$ constitute a first example of the control of intra-molecular electronic dynamics under the influence of the laser phase and thus provide a first clue that intra-molecular electron transfer processes may be controllable by light fields of controlled evolution.

\section{Acknowledgement}

We acknowledge contributions by Y. Ni, J.I. Khan, M. Schultze, T. Uphues, J. Rauschenberger, M. Uiberacker and M. Drescher to these studies. We thank the European Union for support by the Marie Curie Research Training Network XTRA, MRTN-CT-2003-505138, a Marie Curie Intra-European Fellowship, MEIF-CT-2003-500947, and a European Reintegration Grant. The research of M.F.K., C.S. and M.J.J.V. is 
part of the research program of the "Stichting voor Fundamenteel Onderzoek der Materie (FOM)", which has been financially supported by the "Nederlandse Organisatie voor Wetenschappelijk Onderzoek (NWO). M.F.K., I.Z. and S.Z. acknowledge support by the Max-Planck Society and by the German Science Foundation via the Emmy-Noether program. This work was partly supported by the Cluster of Excellence "Munich Center for Advanced Photonics" (MAP).

\section{Figures and Captions}

Figure 1: A) Schematic view of the velocity map imaging experiment. Laser pulses are CEP controlled using a pair of wedges. The beam is focused with a spherical mirror $(\mathrm{f}=40 \mathrm{~cm})$ into the center of the ion optics, where it crosses a molecular beam. Resulting ions are extracted and analyzed using a dual microchannel and phosphor screen detector. A typical velocity map image from the detection of $\mathrm{D}^{+}$ions in the dissociation of $\mathrm{D}_{2}$ with 5 fs pulses at $10^{14} \mathrm{~W} \mathrm{~cm}^{-2}$ without phase stabilization is shown in $\mathrm{B}$ ).

Figure 2: A) $\mathrm{D}^{+}$kinetic energy spectrum from the interaction of $\mathrm{D}_{2}$ with 5 fs linear and circular polarized laser pulses at $10^{14} \mathrm{~W} \mathrm{~cm}^{-2}$ without phase stabilization $\mathrm{B}$ ) Angular distributions for $\mathrm{D}^{+}$from $\mathrm{D}_{2}$ within three energy windows corresponding to the BS $(0-2 \mathrm{eV})$, EI $(2-3 \mathrm{eV})$ and RCE $(3-8 \mathrm{eV})$ channels as measured for the conditions in A) for linear polarization. C) Schematic diagram showing the different dissociation pathways that yield $\mathrm{D}^{+}$ions from $\mathrm{D}_{2}$ via dissociation of the molecular ion via recollisional excitation (RCE) or coulomb explosion of $\mathrm{D}_{2}{ }^{2+}$ in enhanced ionization (EI); in strong laser fields, bond softening (BS) may lead to dissociation of the molecular ion, where the avoided crossing between diabatic potentials that are dressed by the laser field (as an example, the $2 p \sigma_{u}{ }^{+}$potential dressed with (-1) photon is drawn as a dashed line) results in an energy gap that gives rise to dissociation from vibrational levels that were originally bound [35]. BS was studied in great detail for different light intensities and pulse durations [47]. Note that further channels playing a role at higher intensities than in the present studies are omitted in the scheme.

Figure 3: A) $D^{+}$kinetic energy spectrum with 5 fs linear laser pulses at $10^{14} \mathrm{~W} \mathrm{~cm}^{-2}$ without phase stabilization. B) Map of asymmetry parameter $A(W, \theta)$ as a function of the $\mathrm{D}^{+}$kinetic energy $W$ and the carrier envelope phase $\varphi_{\text {CEP }}$ (measured over a range of $6 \pi$ with a step size of $0.1 \pi$ ). C) Asymmetry integrated over several energy ranges versus the CEP. D) Degree of alignment of D+ ions (represented by the expectation value of $\left.\cos ^{2}(\theta)\right)$ versus the CEP for the same energy intervals as in C).

Figure 4: A) Maximum degree of asymmetry $\mathrm{A}_{0}(W, \theta)$ in the emission of $\mathrm{D}^{+}$ions from the dissociative ionization of $\mathrm{D}_{2}$ as a function of the emission angle and energy. The phase dependent asymmetry oscillations have been fit to sine functions $A\left(W, \theta, \varphi_{\mathrm{CEP}}\right)=A_{0}(W, \theta) \sin \left(\varphi_{\mathrm{CEP}}+\varphi_{0}(W, \theta)\right)$ to obtain the parameter $\mathrm{A}_{0}(W, \theta)$. The effect is limited to $\theta= \pm 50^{\circ}$. The butterfly shape of the effect indicates two different mechanisms, based on RCE for high energies and BS for small ion energies (see text). B) Experimental dependence of the asymmetry modulation depth in the emission of $\mathrm{D}^{+}$ions between 3 and $8 \mathrm{eV}$ from dissociation of $\mathrm{D}_{2}$ on the laser pulse duration.

Figure 5: Results of the simulations: About 2/3 of an optical cycle after an electron has been liberated from the neutral molecule (accompanied by the production of a wave packet evolves along the ground ionic state $1 \mathrm{~s} \sigma_{\mathrm{g}}{ }^{+}$), this electron recollides with the parent and excites the part of the population that is relevant for the explanation of the experimental results to the $2 \mathrm{p \sigma}_{\mathrm{u}}^{+}$state. A superposition of both the $1 \mathrm{~s} \sigma_{\mathrm{g}}{ }^{+}$and the $2 \mathrm{p \sigma}_{\mathrm{u}}^{+}$ 
states is formed in the laser field by population transfer. This breaks the parity of the electronic wavefunction, and allows to control the final localization of the charge on the "left" and the "right" part of the molecule.

Figure 6: Asymmetry data obtained for the case of $\mathrm{HD}, \mathrm{D}^{+}$ions. A) Sample image and kinetic energy spectrum for $\mathrm{D}^{+}$ions from the dissociation of $\mathrm{HD}$ with $5 \mathrm{fs}, 10^{14} \mathrm{Wcm}^{-2}$ pulses. B) Map of the asymmetry parameter $A(W, \theta)$ as a function of the $\mathrm{D}^{+}$kinetic energy $W$ and phase $\varphi_{\text {CEP. }}$ C) Asymmetry parameter integrated over selected energy ranges (as indicated) versus the CEP.

Figure 7: Asymmetry data obtained for the case of $\mathrm{HD}, \mathrm{H}^{+}$ions. A) Sample image and kinetic energy spectrum for $\mathrm{H}^{+}$ions from the dissociation of $\mathrm{HD}$ with $5 \mathrm{fs}, 10^{14} \mathrm{Wcm}^{-2}$ pulses. B) Map of the asymmetry parameter $A(W, \theta)$ as a function of the $\mathrm{H}^{+}$kinetic energy $W$ and phase $\varphi_{\text {CEP. }}$ C) Asymmetry parameter integrated over selected energy ranges versus the CEP.

Figure 8: Maximum degree of asymmetry $\mathrm{A}_{0}(W, \theta)$ in the emission of $\mathrm{D}^{+}$ions from the dissociative ionization of $\mathrm{HD}$ as a function of the emission angle and energy. The phase dependent asymmetry oscillations have been fit to sine functions $A\left(W, \theta, \varphi_{\mathrm{CEP}}\right)=A_{0}(W, \theta) \sin \left(\varphi_{\mathrm{CEP}}+\varphi_{0}(W, \theta)\right)$ to obtain the parameter $\mathrm{A}_{0}(W, \theta)$.

Figure 9: A) Spectrum and B) asymmetry map $A\left(W, \varphi_{\text {CEP }}\right)$ for the emission of electrons in above-threshold ionization of Xe with $5 \mathrm{fs}$ pulses at $10^{14} \mathrm{~W} \mathrm{~cm}^{-2}$. The phase was set to zero at the maximum asymmetry for cut-off electrons and used to calibrate the phase axis in figures 6 and 7.

\section{References}

1. Lenzner, M., et al., 1998, Extreme nonlinear optics with few-cycle laser pulses. IEICE Transactions, E81-C(2): p. 112-122.

2. Baltuska, A., et al., 2003, Attosecond control of electronic processes by intense light fields. Nature, 421(6923): p. 611-615.

3. Lewenstein, M., et al., 1994, Theory of High-Harmonic Generation by Low-Frequency Laser Fields. Phys. Rev. A, 49(3): p. 2117-2132.

4. Li, X.F., et al., 1989, Multiple-harmonic generation in rare gases at high laser intensity. Phys. Rev. A, 39(11): p. 5751-5761.

5. Freeman, R.R., et al., 1987, Above-threshold ionization with subpicosecond laser pulses. Phys. Rev. Lett., 59(10): p. 1092-1095.

6. Paulus, G.G., et al., 1994, Plateau in above threshold ionization spectra. Phys. Rev. Lett., 72(18): p. 2851-2854.

7. Spanner, M., et al., 2004, Reading diffraction images in strong field ionization of diatomic molecules. J. Phys. B, 37(12): p. L243-L250.

8. Itatani, J., et al., 2004, Tomographic imaging of molecular orbitals. Nature, 432(7019): p. 867-871.

9. Paulus, G.G., et al., 2003, Measurement of the phase of few-cycle laser pulses. Phys. Rev. Lett., 91(25): p. 253004.

10. Liu, X., et al., 2004, Nonsequential double ionization at the single-optical-cycle limit. Phys. Rev. Lett., 93(26): p. 263001.

11. Reichert, J., et al., 1999, Measuring the frequency of light with mode-locked lasers. Opt. Comm., 172(1-6): p. 59-68.

12. Goulielmakis, E., et al., 2004, Direct Measurement of Light Waves. Science, 305: p. 1267-1269. 
13. Kienberger, R., et al., 2004, Atomic transient recorder. Nature, 427(6977): p. 817-821.

14. Lindner, F., et al., 2005, Attosecond double-slit experiment. Phys. Rev. Lett., 95(4): p. 040401.

15. Kling, M.F., et al., 2006, Control of electron localization in molecular dissociation. Science, 312(5771): p. 246-248.

16. Sansone, G., et al., 2006, Isolated single-cycle attosecond pulses. Science, 314(5798): p. 443-446.

17. Uiberacker, M., et al., 2007, Attosecond real-time observation of electron tunnelling in atoms. Nature, 446(7136): p. 627-632.

18. Cavalieri, A.L., et al., 2007, Attosecond spectroscopy in condensed matter. Nature, 449: p. 1029-1032.

19. Posthumus, J.H., 2004, The dynamics of small molecules in intense laser fields. Rep. Progr. Phys., 67(5): p. 623-665.

20. Alnaser, A.S., et al., 2005, Simultaneous real-time tracking of wave packets evolving on two different potential curves in H-2(+) + and D-2(+). Phys. Rev. A, 72(3): p. 030702.

21. Tong, X.M. and C.D. Lin, 2007, Dynamics of Light-Field Control of Molecular Dissociation at the Few-Cycle Limit. Phys. Rev. Lett., 98: p. 123002.

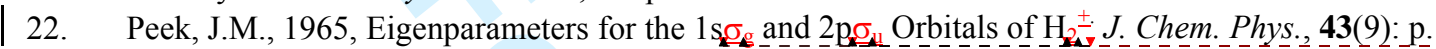
I 3004-3006.

23. Kolos, W., K. Szalewicz, and H.J. Monkhorst, 1986, New Born--Oppenheimer potential energy curve and vibrational energies for the electronic ground state of the hydrogen molecule. J. Chem. Phys., 84(6): p. 3278-3283.

24. Bandrauk, A.D., S. Chelkowski, and H.S. Nguyen, 2004, Attosecond localization of electrons in molecules. Int. J. Quant. Chem., 100(6): p. 834-844.

25. Gräfe, S. and M. Ivanov, 2007, Effective Fields in Laser-Driven Electron Recollision and Charge Localization. Phys. Rev. Lett., 99: p. 163603.

26. Haljan, P., M.Y. Ivanov, and P.B. Corkum, 1997, Laser control of electron localization in molecules

| and double quantum wells. Laser Phys., 7(3): p. 839-843.

27. Cavalieri, A.L., et al., 2007, Intense 1.5-cycle near infrared laser waveforms and their use for the generation of ultra-broadband soft-x-ray harmonic continua. New J. Phys., 9(7): p. 242.

28. Rauschenberger, J., et al., 2006, Carrier-envelope phase-stabilized amplifier system. Laser Phys. Lett., 3(1): p. 37-42.

29. Verhoef, A.J., et al., 2006, Few-cycle carrier envelope phase-dependent stereo detection of electrons. Opt. Lett., 31(23): p. 3520-3522.

30. Lepine, F., et al., 2004, Atomic photoionization processes under magnification. Phys. Rev. A, 70: p. 033417.

31. Vrakking, M.J.J., 2001, An iterative procedure for the inversion of two-dimensional ion/photoelectron imaging experiments. Rev. Sci. Instr., 72(11): p. 4084-4089.

32. Niikura, H., et al., 2002, Sub-laser-cycle electron pulses for probing molecular dynamics. Nature, 417(6892): p. 917-922.

33. Niikura, H., et al., 2003, Probing molecular dynamics with attosecond resolution using correlated wave packet pairs. Nature, 421(6925): p. 826-829.

34. Alnaser, A.S., et al., 2004, Routes to control of H-2 Coulomb explosion in few-cycle laser pulses. Phys. Rev. Lett., 93(18): p. 183202.

35. Bucksbaum, P.H., et al., 1990, Softening of the H2+ Molecular Bond in Intense Laser Fields. Phys. Rev. Lett., 64(16): p. 1883-1886.

36. Niikura, H., P.B. Corkum, and D.M. Villeneuve, 2003, Controlling vibrational wave packet motion with intense modulated laser fields. Phys. Rev. Lett., 90(20): p. 203601. 
37. Ergler, T., et al., 2006, Spatiotemporal imaging of ultrafast molecular motion: Collapse and revival of the D2+ nuclear wave packet. Phys. Rev. Lett., 97(19): p. 193001.

38. Ammosov, M.V., N.B. Delone, and V.P. Krainov, 1986, Sov. Phys. JETP, 64: p. 1191.

39. Roudnev, V. and B.D. Esry, 2007, $\mathrm{HD}_{+}^{+}$in a short strong laser pulse: Practical consideration of the observability of carrier-envelope phase effects, Phys. Rev. A, 76: $\bar{p} .023403$,

40. Kling, M.F., et al., 2007, Imaging of carrier-envelope phase effects in above-threshold ionization with intense few-cycle laser fields. New J. Phys., submitted.

41. Goulielmakis, E., et al., 2007, Attosecond control and measurement: Lightwave electronics. Science, 317(5839): p. 769-775.

42. Remacle, F. and R.D. Levine, 2006, An electronic time scale in chemistry. Proc. Nat. Acad. Sci. USA,

I 43. 103(18): p. 6793-6798. Phys. Rev. Lett., 94: p. 033901.

44. Niikura, H., et al., 2005, Attosecond dynamics using sub-laser-cycle electron pulses. J. Mod. Opt., 52(2-3): p. 453-464.

45. Yudin, G.L., et al., 2005, Attosecond photoionization of coherently coupled electronic states. Phys. Rev. A, 72(5): p. 051401.

46. Barth, I. and J. Manz, 2006, Periodic Electron Circulation Induced by Circularly Polarized Laser Pulses: Quantum Model Simulations for Mg-porphyrin. Angew. Chem. Intern. Ed., 45: p. 2962-2965.

47. Sändig, K., H. Figger, and T.W. Hänsch, 2000, Dissociation Dynamics of H2+ in Intense Laser Fields: Investigation of Photofragments from Single Vibrational Levels. Phys. Rev. Lett., 85(23): p. 48764879 . 
Figure 1
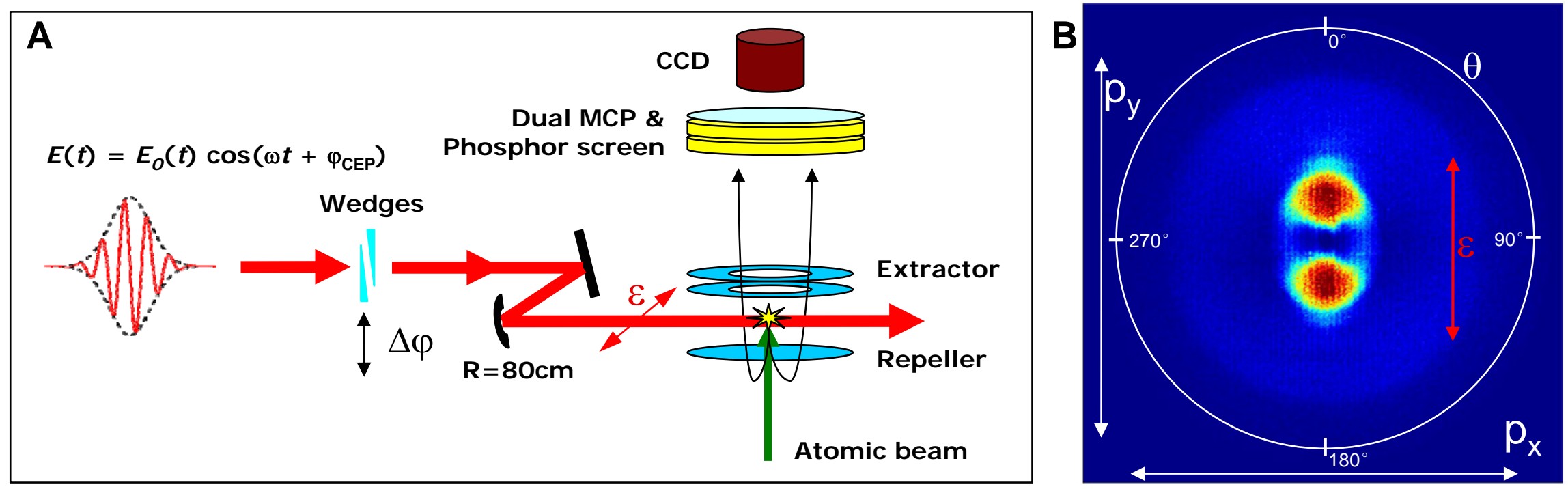
Figure 2
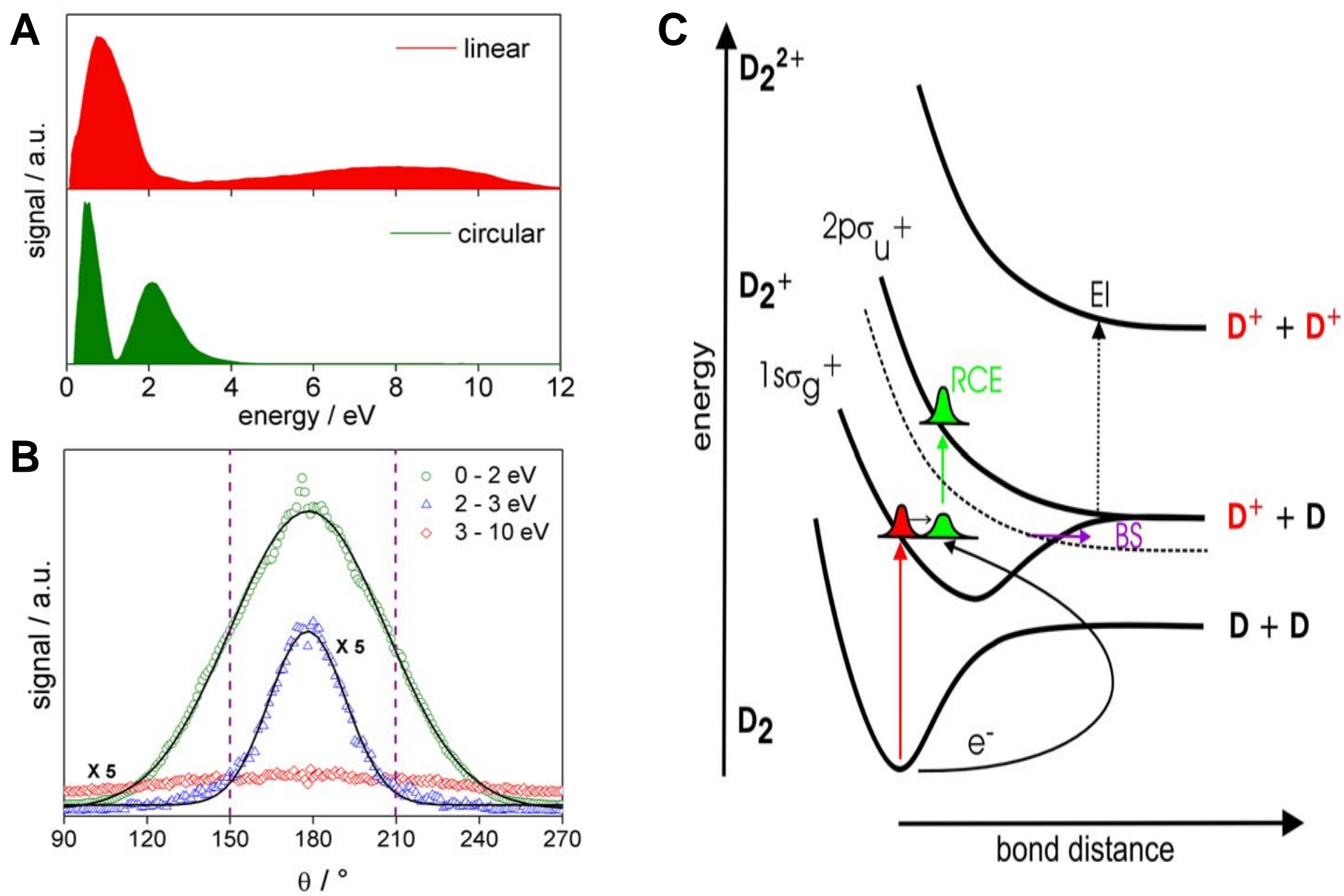
Figure 3

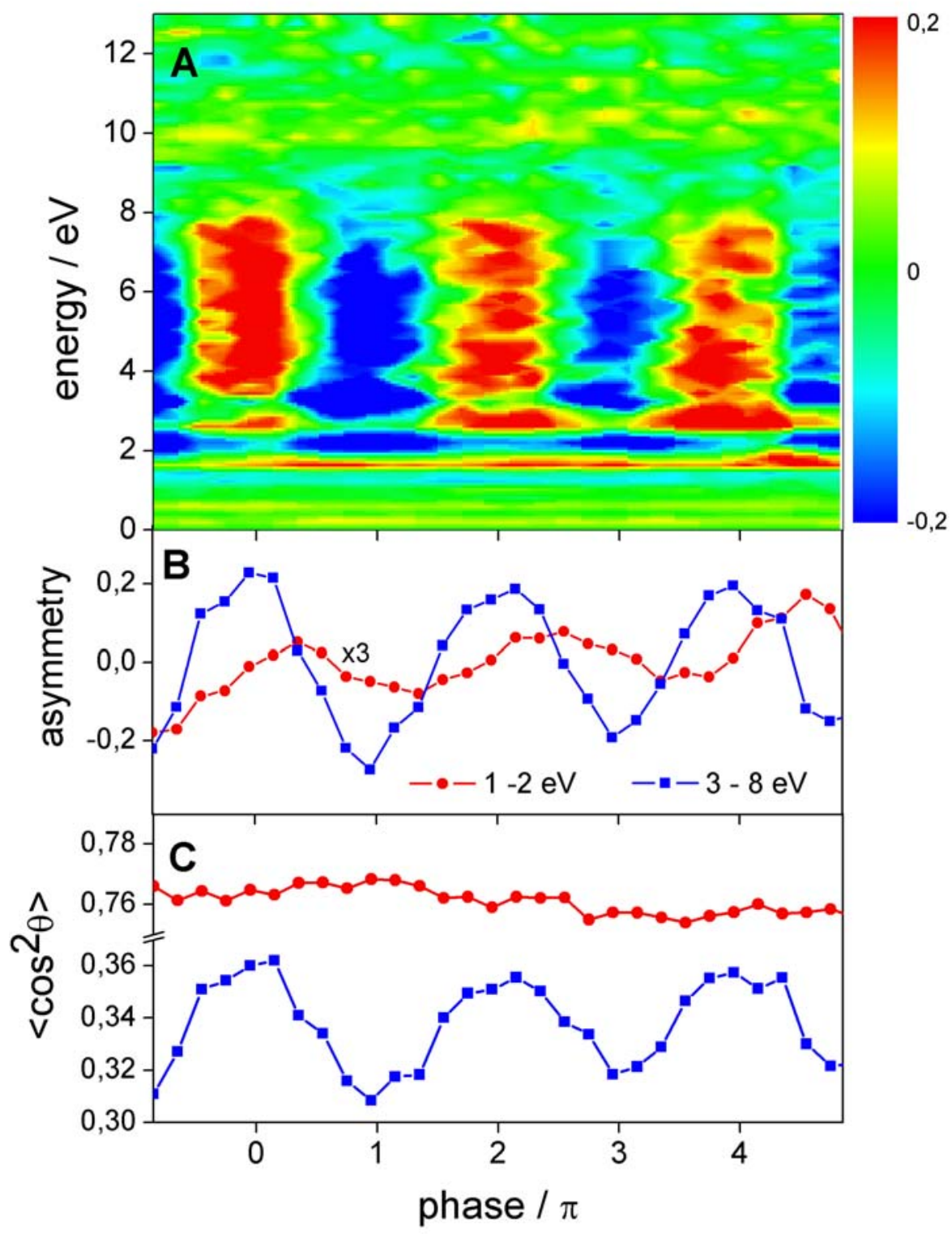

URL: http://mc.manuscriptcentral.com/tandf/tmph 


\section{Figure 4}
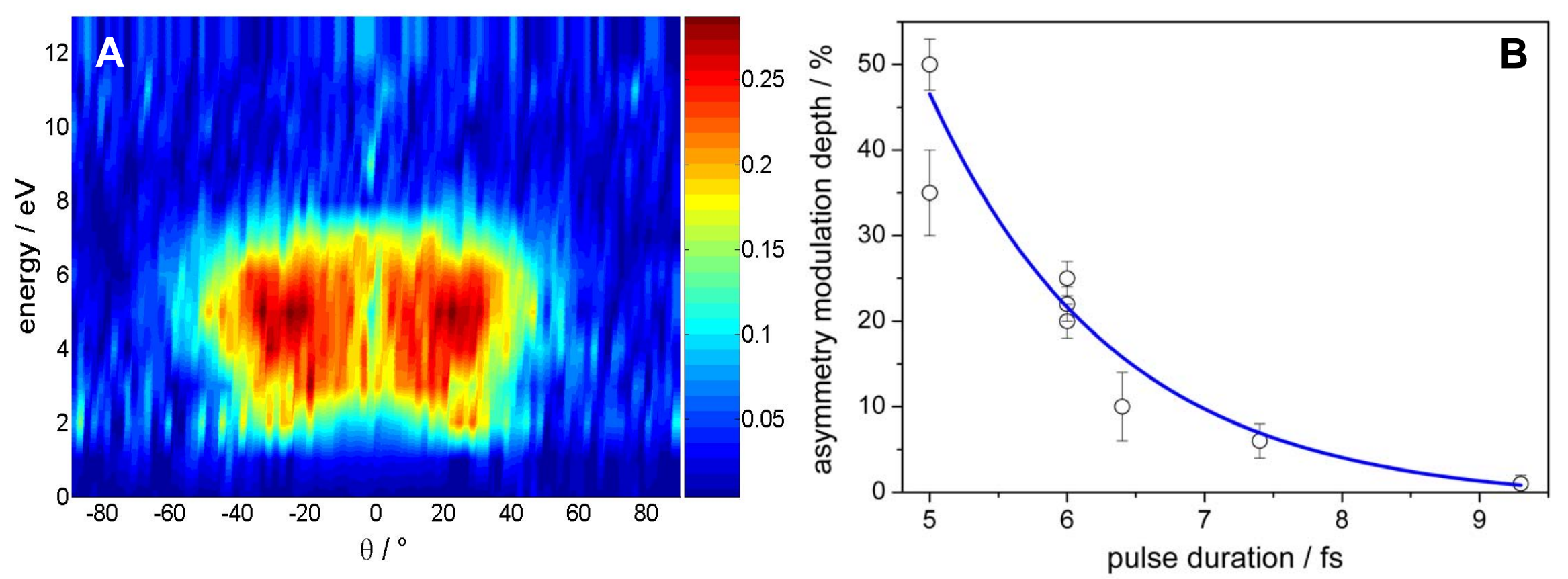


\section{Figure 5}

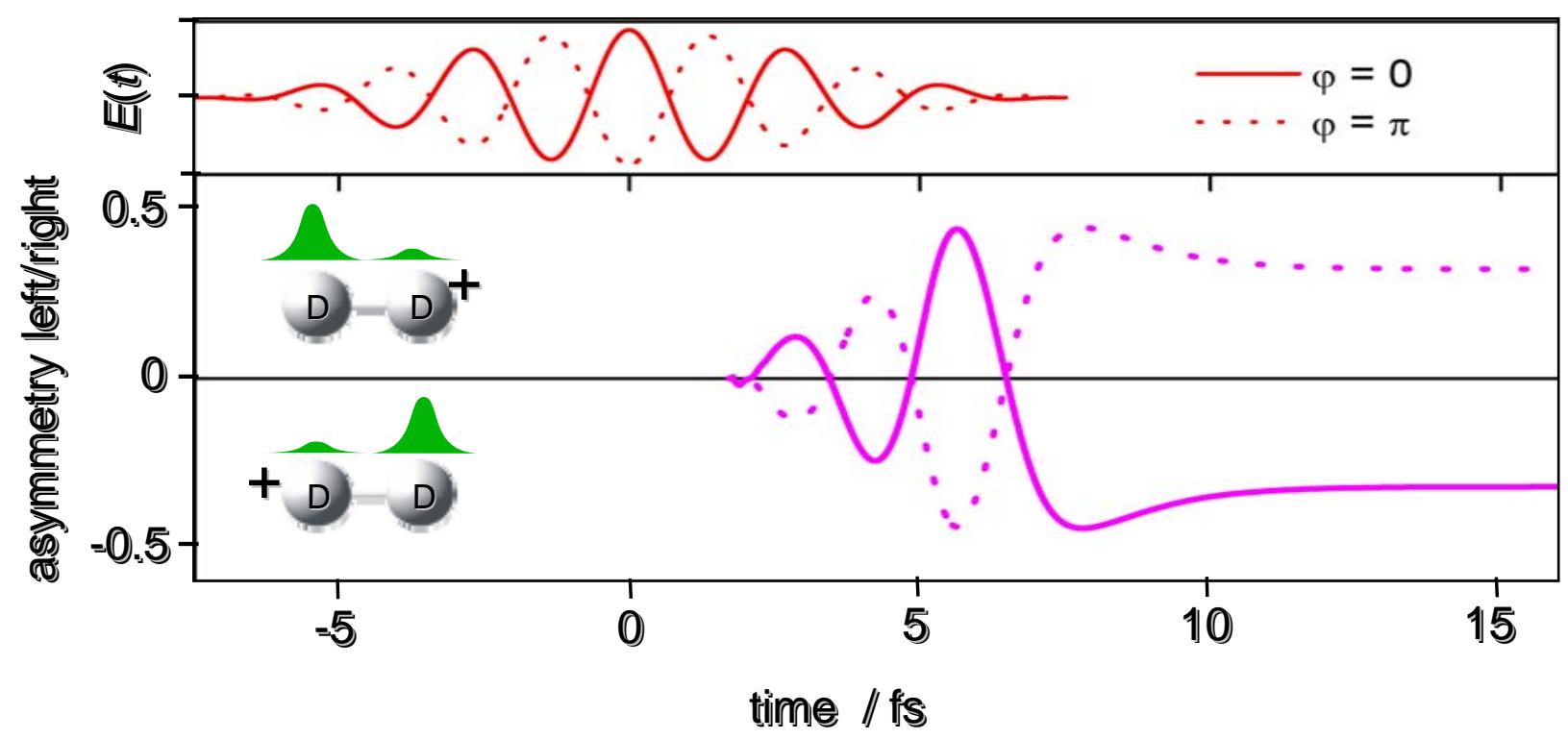




\section{Figure 6}

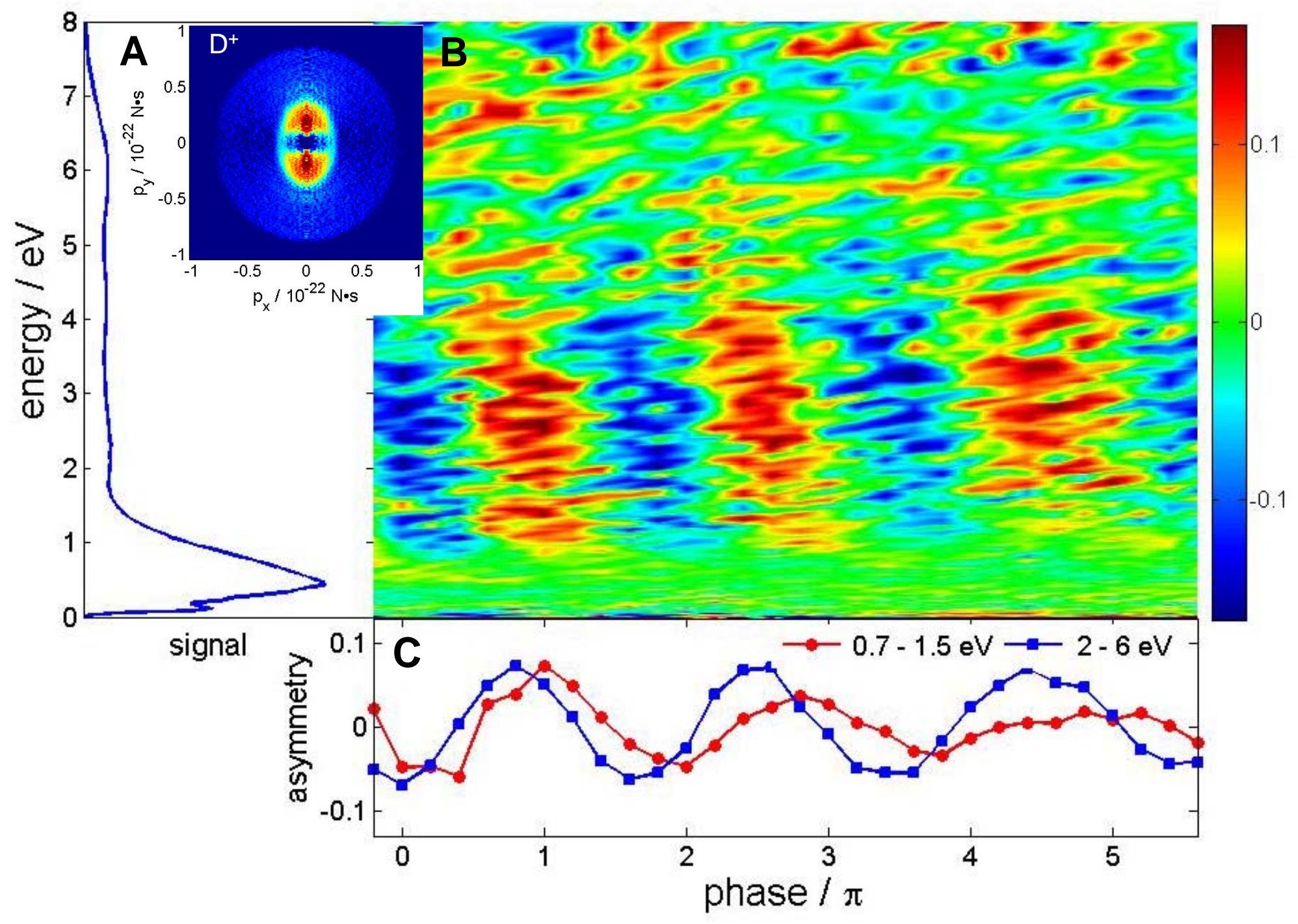




\section{Figure 7}

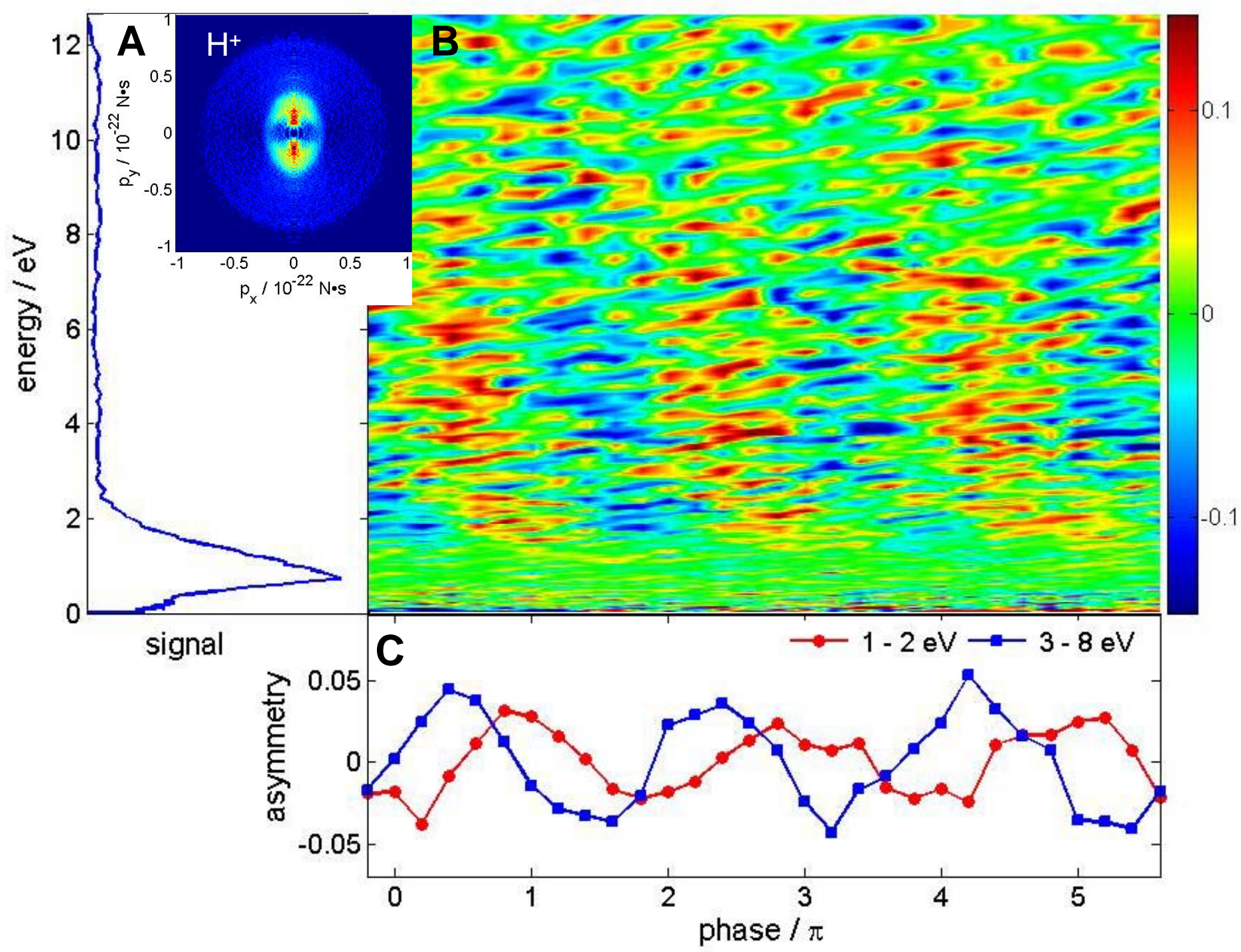


Figure 8

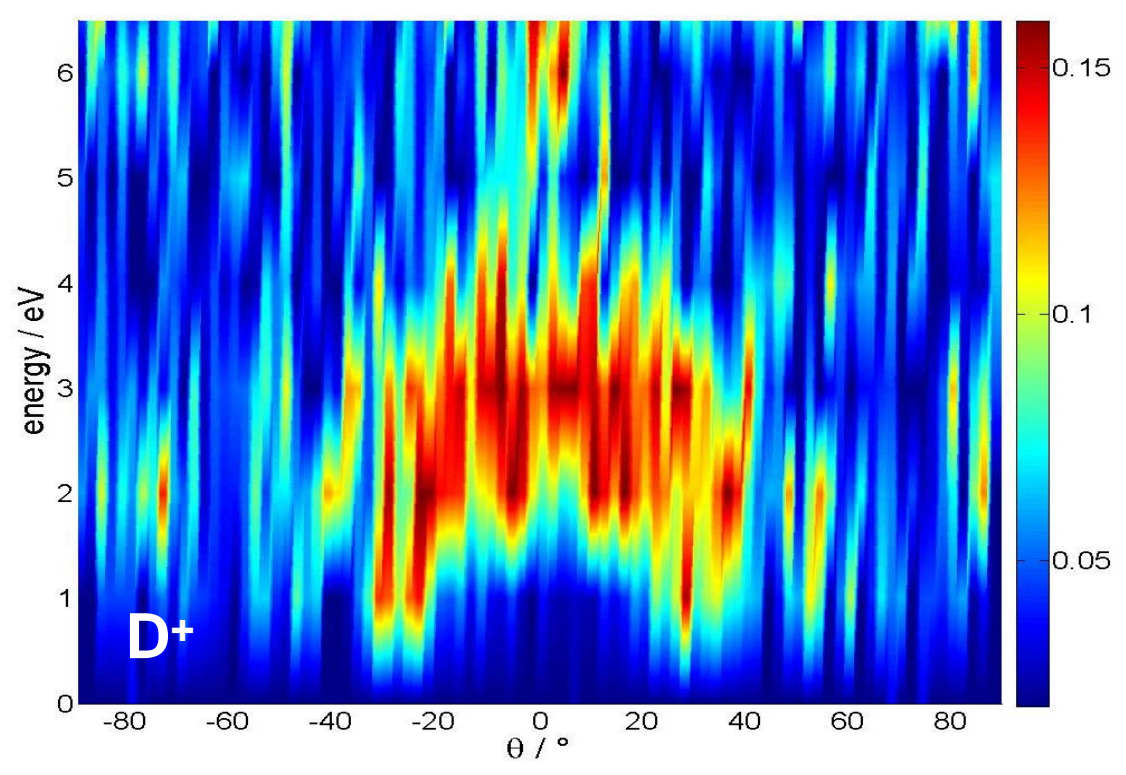




\section{Figure 9}

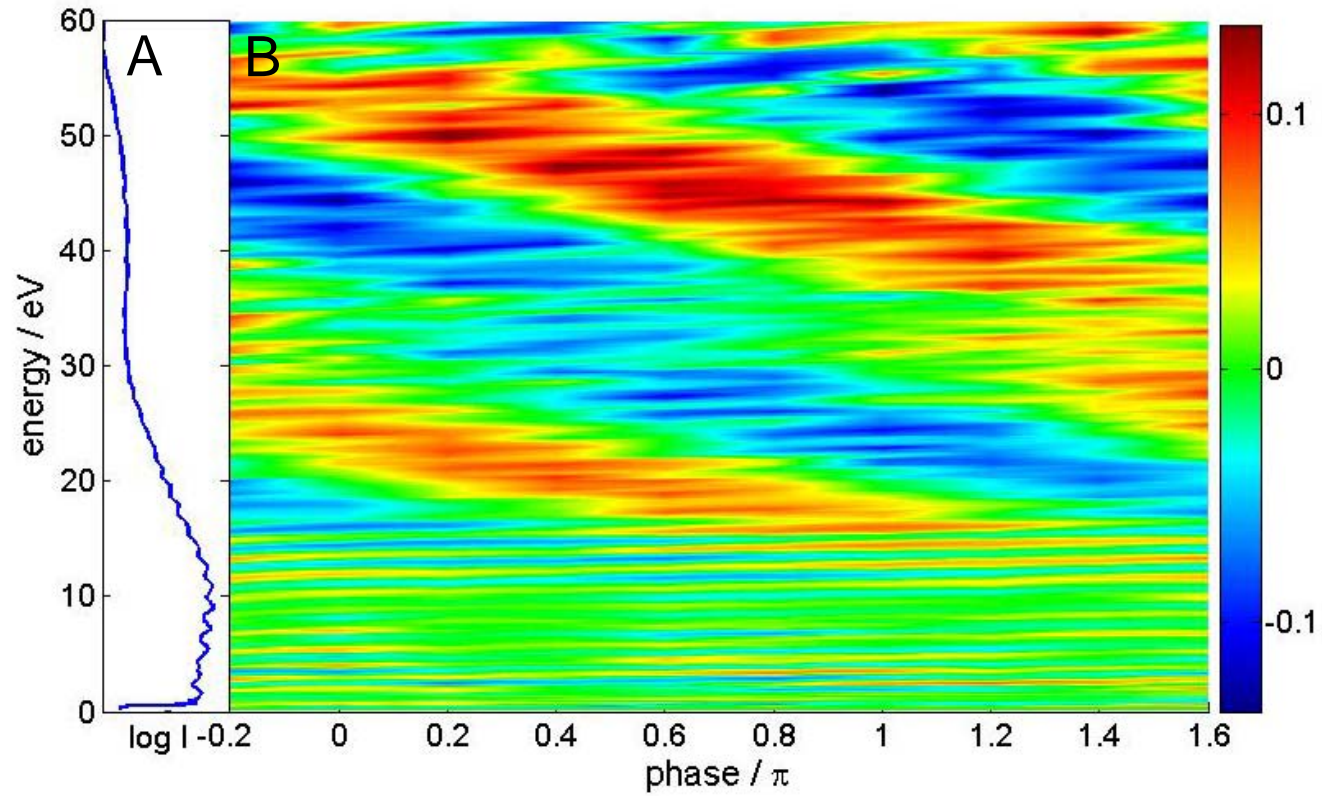


Catchline (head of first page only) Molecular Physics, Vol. X, No. X, Month 2008, xxx-xxx

Running heads (verso) M.F. Kling et al.

(recto) Strong-field control of electron localization during molecular

dissociation

Article Type (e.g. Research Article)

\title{
Strong-field control of electron localization during molecular dissociation
}

\author{
M.F. Kling ${ }^{1,2^{*}}$, Ch. Siedschlag ${ }^{1}$, I. Znakovskaya, ${ }^{2}$ A.J. Verhoef, ${ }^{2}$ S. Zherebtsov, ${ }^{2}$ F. Krausz, ${ }^{2,3}$ M. Lezius, ${ }^{2}$ \\ M.J.J. Vrakking ${ }^{1 *}$ \\ ${ }^{1}$ FOM Instituut voor Atoom en Molecuul Fysica (AMOLF), Kruislaan 407, 1098 SJ Amsterdam, Netherlands \\ ${ }^{2}$ Max-Planck-Institut für Quantenoptik, Hans-Kopfermann-Strasse 1, D-85748 Garching, Germany \\ ${ }^{3}$ Department für Physik, Ludwig-Maximilians-Universität München, Am Coulombwall 1, D-85748 Garching, \\ Germany \\ *E-mails: matthias.kling@mpq.mpg.de, m.vrakking@amolf.nl \\ Dedicated to Prof. Raphy Levine on the occasion of his 70th anniversary.
}

\begin{abstract}
We demonstrate how the waveform of light can be used to control a molecular dissociation by steering and localization of electrons. Experimental results have been obtained for the dissociative ionization of the homonuclear and heteronuclear Hydrogen derivates $\mathrm{D}_{2}$ and HD. Asymmetric ejection of the ionic fragments reveals that light-driven electronic motion prior to dissociation localizes the electron on one of the two ions in the diatomic molecular ions in a controlled way. Extension of these results to electron transfer in complex molecules suggests a new paradigm for controlling photochemistry.
\end{abstract}

Keywords: Strong-field control, few cycle laser pulses, charge localization AMS Subject Classification: 81V55; 81 V80 


\section{Introduction}

Coherent control of molecular dynamics has entered a new and exciting regime with the advent of intense few-cycle phase stabilized laser pulses [1]. Laser technology now allows for the generation and control of electromagnetic fields, where the electric field can be switched between 0 and several a.u. with a temporal accuracy of a few 100 as [2]. Obviously, only electrons can respond on this timescale, and atomic centers will remain frozen. If the laser intensity is chosen carefully, the extreme non-linearity of the strong field tunneling probability due to the Gamov-factor $\exp \left(-2\left(2 U_{\mathrm{I}}\right)^{3 / 2} / 3|E(t)|\right)$, with $U_{\mathrm{I}}$ the ionization potential and $E(t)$ the electric field, leads to situations where electrons are liberated from a molecule within a fraction (100-300 as) of the cycle of the carrier wave. A full cycle of this carrier wave typically lasts about 2660 as at $800 \mathrm{~nm}$ when using Ti:Sapphire lasers. Subsequently, these electrons are driven by the laser field [3], which leads to daughter processes that can, in principle, be precisely synchronized with respect to the original ionization event. Typical cases are recombination and high-order harmonic generation [4], scattering and high energy above-threshold ionization (ATI) [5, 6], as well as attosecond electron diffraction [7]. All of these processes take place about $1 / 3$ of the laser cycle after strong field ionization (SFI) has happened close to the peak electric field [3], when the electron revisits its parent near a zero-crossing of the electric field. The electron rescattering process can also lead to population transfer into excited states above the ionic ground state, which is usually prepared during the strong field tunneling process. This is especially attractive to molecular physics, because the preparation of higher excited molecular states can thus be very precisely timed. Furthermore, because electron rescattering is approximately equal to classical electron impact excitation of ions, no strong selection rules apply, in contrast with the optical case. Only if during rescattering recombination takes place, will the molecule preferentially end up in its initial state, and excess energy will be given away as harmonic radiation. This has been used with advantage for the prominent tomographic imaging of molecular orbitals by Itatani et al. [8].

Molecular electron rescattering physics can and has been investigated intensively by various groups in recent years with multi-cycle laser pulses. However, for the case that the laser pulse duration approaches the optical period one enters the few-cycle regime and the electromagnetic driver fields become increasingly asymmetric. Such fields have recently opened up new avenues for coherent control. Spatial control of electron emission has been observed and has become a major tool for long term stabilization of the laser phase [9]. It has also been possible to control total fragment particle momenta [10]. The prerequisite for such experiments, control of the carrier-envelope phase (CEP) itself has become available as a laser control parameter since the groundbreaking work of T. Hänsch and coworkers [11], and its extension towards amplified laser systems by A. Baltuska et al. [2]. The latter has paved the way into the strong field community. Stabilization and control of the laser phase with comparably high precision has made many experiments possible that are directly related to attosecond physics [12-18]. The relation between the CEP and attosecond physics itself can be easily understood, since control over the CEP is virtually equal to control of a light field with attosecond precision. CEP control applied to the few-cycle regime, however, enables access to mono-cycle strong field ionization. In such cases, subsequent steering of isolated attosecond electron wavepackets is feasible and gives access to controlled time dependent and intense polarization of the target system. In combination with molecular alignment or orientation selection via SFI the technique can be used to control the final localization of charge during the molecular dissociation, as has been previously demonstrated for the D-D homonuclear dimer [15]. In this paper, we extend the discussion of such experiments towards the heteronuclear dimer H-D, and towards additional aspects in the CEP control of charge redistribution, which may be attributed to phase control of bond-softening processes. 
Hydrogen ionization and dissociation has been attractive to the femtosecond community for several years (see e.g. [19] and references therein). Some reasons for this are: First, $\mathrm{H}_{2}$ intranuclear vibrational wavepacket dynamics is very fast and requires a temporal resolution in the few-fs regime [20]. Second, because only two electrons and two protons are involved, the system can be numerically accessed with high accuracy [21]. As such, it has model character for the treatment of more complex molecules and with regard to chemistry perhaps more interesting cases. Third, lower ionic levels in Hydrogen are energetically well separated [22, 23]. Because of this, IR multiphoton ionization ends up mostly in one single electronic state $\left(1 \mathrm{~s} \sigma_{\mathrm{g}}{ }^{+}\right)$. Subsequent electron rescattering events then populate a superposition of higher states, e.g. create a synchronized electronic wavepacket. The corresponding coupled electron-nuclear dynamics evolving within the rapidly decaying strong laser field can be made responsible for final charge localization [15, 24-26].

\section{Experimental}

The experimental scheme that was used here has been described earlier [15]. In brief, transform-limited laser pulses of 25 fs duration with $1 \mathrm{~mJ}$ pulse energy have been generated with a $3 \mathrm{kHz}$ phase stabilized amplified Ti:sapphire laser system (Femtolasers, Femtopower Compact Pro). The pulse was spectrally broadened using a one-meter long hollow-core fiber of $250 \mu \mathrm{m}$ diameter filled with 3.8 bar Neon gas. The laser pointing into the fiber was controlled with high precision with a home-built stabilizer system consisting of a CCD camera and a motorized mirror mount. The output pulses from the fiber exhibited a significantly broadened spectrum (see e.g. [27]) and were compressed down to a near-transform limited duration of $\sim 5 \mathrm{fs}$ using 8 reflections in a chirped mirror compressor. By tuning of the gas pressure in the hollow-core fiber the pulse length was adjusted precisely between 25 and 5 fs. The pulse duration was monitored online with a commercial dispersion balanced autocorrelator. The laser phase has been stabilized with a feedback loop described in [28, 29]. The polarization is rectified using three $5 \mu \mathrm{m}$ thick pellicles at Brewster angle. Fine tuning of the pulse duration and varying the CEP was done by changing the amount of material dispersion with a pair of fused silica wedges after the hollow fibre. The phase jitter was smaller than $150 \mathrm{mrad}$. Phase-stabilized, linearly polarized pulses at a central wavelength of $760 \mathrm{~nm}$ were focused with a spherical mirror $(\mathrm{R}=80 \mathrm{~cm})$ into the center of the ion optics of a velocity-map imaging spectrometer [30]. In the focus intensities of up to $5 \cdot 10^{14} \mathrm{~W}$ $\mathrm{cm}^{-2}$ have been realized, and an adjustable iris has been used to vary the intensity in the focus. Ions and electrons were generated at the crossing point between the laser and the molecular beam (with a particle density of appr. $10^{13} \mathrm{~cm}^{-3}$ ) and were accelerated and focused with the ion optics onto a MCP-phosphor screen assembly (Hamamatsu, F2226-24PX). The molecular beam was produced by a pulsed nozzle of $1 \mathrm{~mm}$ diameter and operated at $50 \mathrm{~Hz}$. The molecular beam was differentially pumped and passed a skimmer of 1 $\mathrm{mm}$ diameter $10 \mathrm{~cm}$ downstream before entering the interaction region. The sensitivity of the MCP-detector was switched so that only ions or electrons from every 60th laser pulse were detected. This allowed for a low background pressure in the chamber (typically $2 \cdot 10^{-7}$ mbar). The velocity map images have been recorded with a CCD camera (Pulnix, TM-9701) and were typically averaged over $60 \mathrm{~s}$ of data acquisition for each setting of the laser phase.

Figure $1 \mathrm{~b}$ shows a typical experimental momentum map of $\mathrm{D}^{+}$ions recorded with 5 fs excitation at $10^{14} \mathrm{~W}$ $\mathrm{cm}^{-2}$ without CEP stabilization. The laser was polarized along the $p_{\mathrm{y}}$-axis. Two main contributions are visible in both the upwards and the downwards emission direction: a strong peak at lower energies with a relatively narrow angular distribution and a contribution at larger momenta with a wide angular distribution. After an inversion of the image using an iterative inversion procedure [31], the original 3D-momentum distribution can be reconstructed. By integration of the inverted image over the full solid angle the energy spectrum displayed in Figure $2 \mathrm{~A}$ is derived. The spectral features can be attributed to the following reaction pathways (as sketched in Figure 2C): 
A) Following strong-field tunnel ionization of $\mathrm{D}_{2}$ with the production of an electron and $\mathrm{D}_{2}{ }^{+}$ion in the $1 \mathrm{~s}{\sigma_{\mathrm{g}}}^{+}$-state, recollisional excitation (RCE) $[32,33]$ to the $2 \mathrm{p \sigma}_{\mathrm{u}}{ }^{+}$state by the returning electron leads to dissociation and formation of $\mathrm{D}^{+}$and $\mathrm{D}$ fragments with energies above $3 \mathrm{eV}$. This channel shows a broad angular distribution as seen in Figure 2B in agreement with earlier observations.[34] Note that no ions at these energies are observed with circular polarized light (Fig. 2A), strongly supporting recollision to be responsible for their production.

B) Close to the outer turning point of the nuclear wavepacket bond softening (BS) $[19,35,36]$ becomes a prominent process, leading to very low fragment energies below $3 \mathrm{eV}$ with its main contribution between 0 and $2 \mathrm{eV}$.

C) At the intensities used in these studies, Coulomb explosion of $\mathrm{D}_{2}$ with the production of two $\mathrm{D}^{+}$ions is possible via enhanced ionization (EI). This channel is, however, unwanted for the present investigations and therefore we tried to keep such signals at a minimum. In fact, only a minor contribution of EI is seen between 2 and $3 \mathrm{eV}$, which exhibits a smaller angular distribution than the BS pathway (see Figure 2B).

$\underline{\text { Results I: electron localization in } \mathrm{D}_{2}}$

In order to elucidate the role of the CEP on the experimental ion momentum distributions, the laser phase was scanned carefully over a range of multiple cycles. The angle-integrated asymmetry in the ion momentum distribution at a certain energy $\mathrm{W}=\mathrm{p}^{2} /(2 \mathrm{~m})$ and phase $\varphi$ was obtained from

$A(W, \varphi)=\frac{P_{u p}(W, \varphi)-P_{d o w n}(W, \varphi)}{P_{u p}(W, \varphi)+P_{d o w n}(W, \varphi)}$

with

$P_{\text {up }}(W, \varphi)=\int_{330}^{360} \int_{0}^{360} P(W, \theta, \phi, \varphi) \sin \theta d \theta d \phi+\int_{0}^{30} \int_{0}^{360} P(W, \theta, \phi, \varphi) \sin \theta d \theta d \phi$

and

$P_{\text {down }}(W, \varphi)=\int_{150}^{210} \int_{0}^{360} P(W, \theta, \phi, \varphi) \sin \theta d \theta d \phi$,

with $\theta$ and $\varphi$ being the polar and azimuthal angles, respectively. We chose to analyze the ion emission within a restricted angular range because our ability to control electron motion in hydrogen requires that the laser couples the two lowest-lying electronic states. For molecules aligned orthogonally to the laser polarization axis, this coupling would be absent.

The contour plot in Figure $3 \mathrm{~A}$ shows the measured angle-integrated asymmetry $A\left(W, \varphi_{\mathrm{CEP}}\right)$ for dissociative ionization of $\mathrm{D}_{2}$ into $\mathrm{D}^{+}+\mathrm{D}$ as a function of the carrier-envelope phase $\varphi_{\mathrm{CEP}}(\mathrm{x}$-axis) and the kinetic energy $W$ of the $\mathrm{D}^{+}$ion fragment (y-axis, see also Figure 2). Note that here the laser phase is only given as a relative number as the absolute phase has not been determined. Figure 3A shows that in an energy range between 3 and $8 \mathrm{eV}$ locking of the laser phase in the few-cycle limit causes a remarkable asymmetry in the upward and downward emission. Regions in Figure $3 \mathrm{~A}$ where the asymmetry oscillates as a function of the phase represent final energies of the $\mathrm{D}^{+}$ions where the direction of their emission is effectively controlled by the sub-cycle evolution of the laser field driving the photodissociation. The extent of this ability to control is further illustrated in Fig. 3B, which displays asymmetries that are integrated over selected energy intervals. 
The highest degree of asymmetry, with a modulation depth of up to $45 \%$, is observed between 3 and $8 \mathrm{eV}$. Above $8 \mathrm{eV}$, the asymmetry appears to cease completely. A very small phase dependence is seen between 1 and $2 \mathrm{eV}$ (see also Figure 3B), which represents the typical energy range for bond softening. Most interestingly, this low energy channel for charge localization appears to be out of phase by ca. $\pi / 2$ with respect to the high energy channel. In principle, when using 5 fs laser pulses, bond softening would not be expected, since this process requires motion of the vibrational wave packet that is formed by the tunnel ionization to the outer turning point of the $1 \mathrm{~s}{\sigma_{\mathrm{g}}}^{+}$potential well. This is expected to take about half a vibrational period, i.e. 12 fs in the case of $\mathrm{D}_{2}$ [37]. However, in our experiments the pulse contrast was not ideal. A background present in the few-cycle pulses amounted to ca. $10 \%$ in intensity at times when the internuclear distance moves into the BS region and might contribute to the asymmetry [26].

Plots of the expectation value of the alignment parameter $\left\langle\cos ^{2}(\theta)\right\rangle$ versus the phase $\varphi_{\mathrm{CEP}}$ are given in Fig. $3 \mathrm{C}$ and show the degree of alignment of $\mathrm{D}^{+}$ions within the energy ranges used in Fig. 3B. A strong phase dependence is again seen for the energy range between 3 and $8 \mathrm{eV}$. This phase dependence is not present in the low energy contribution. A comparison of Figs. 3B and 3C indicates that the observation of a large asymmetry between 3 and $8 \mathrm{eV}$ correlates with a higher degree of alignment. The relation between the charge localization process and the angular distribution of the fragment ions is further explored in Figure 4A, where the amplitude $A_{0}(W, \theta)$ of the asymmetry oscillation $A\left(W, \theta, \varphi_{\mathrm{CEP}}\right)=A_{0}(W, \theta) \sin \left(\varphi_{\mathrm{CEP}}+\varphi_{0}(W, \theta)\right)$ is shown as a function of kinetic energy $\mathrm{W}$ and fragment angle $\theta$. Clearly, for fragment angles $\theta>50$ degrees the asymmetry vanishes. Moreover, a butterfly shape of the asymmetry amplitude is apparent and indicates different mechanisms for the generation of the asymmetry at low and high energies. This will be discussed in more detail below. Additionally, Fig. 4B shows the asymmetry dependence on the pulse duration, which declines quasi-exponentially from ca. $45 \%$ close to 5 fs towards $1 \%$ above 9 fs. This behavior reveals that the asymmetry of the field is driving the charge localization process and that few-cycle pulses are an important prerequisite for the electron localization control that has been achieved.

\section{Theoretical interpretation of charge localization effect in $\mathrm{D}_{2}$}

The electron localization control in the fragmentation of $\mathrm{D}_{2}$ arises due to a phase control mechanism that consists of two parts. The first part has already been partially discussed in relation to the results shown in Figure 2A. The absence of fragments in the kinetic energy range between 3 and $8 \mathrm{eV}$ (where the observed asymmetry is most pronounced) in experiments using circularly polarized light strongly suggests the involvement of a re-collision of the electron that is ejected in the tunnel ionization that produces the $\mathrm{D}_{2}^{+}$ion. Our interpretation is that re-collision of this electron with the $\mathrm{D}_{2}^{+}$ion leads to excitation of the $\mathrm{D}_{2}^{+}$ion from the $1 \mathrm{~s} \sigma_{\mathrm{g}}{ }^{+}$-state to the dissociative $2 \mathrm{p \sigma}_{\mathrm{u}}{ }^{+}$state. Further, indirect support for this is provided by the observation of $\mathrm{D}^{+}$fragments with a kinetic energy of up to $12 \mathrm{eV}$, consistent with acceleration along a repulsive curve starting from an internuclear distance close or equal to the internuclear distance in the neutral ground state. However, this re-collision excitation by itself is not enough to cause an asymmetry. If the observation of a high kinetic energy $\mathrm{D}^{+}$fragment could be used as an indication of the fact that a fragment had been detected that was dissociating along the $2 \mathrm{p \sigma}_{\mathrm{u}}^{+}$potential curve, then the molecule would retain its parity up to the point of detection, and - parity being a symmetry property of the electronic wave function - the distribution of the electron over the two $\mathrm{D}^{+}$ions involved in the dissociation would necessarily have to be symmetric. A second ingredient is required, which breaks the parity of the electronic wave function. As proposed in ref. [15], and subsequently confirmed in more detailed theoretical treatments [21, 25] laser-induced coupling between the $2 \mathrm{p}_{\mathrm{u}}{ }^{+}$state and the $1 \mathrm{~s} \sigma_{\mathrm{g}}{ }^{+}$-state can convert the dissociative wave packet that starts out on the $2 \mathrm{p} \sigma_{\mathrm{u}}{ }^{+}$state into a 
coherent superposition state containing contributions from both the $2 \mathrm{p} \sigma_{\mathrm{u}}{ }^{+}$state and the $1 \mathrm{~s} \sigma_{\mathrm{g}}{ }^{+}$-state and importantly - with a broken parity.

According to the simplified semiclassical model presented in [15] the time evolution of the wave function for the Hydrogen molecule after recollision can be calculated by expanding the full wave function for the electronic coordinate and the internuclear distance in terms of the lowest lying electronic states,

$$
\Psi(\vec{r}, R ; t) \approx|g\rangle \psi_{g}(R ; t)+|u\rangle \psi_{u}(R ; t)
$$

where $|g\rangle$ and $|u\rangle$ correspond to the $1 s \sigma_{g+}$ and the $2 p \sigma_{u+}$ states, respectively, and where $\psi_{g / u}$ represent the corresponding nuclear wave packets. In accordance with our observation that the asymmetry is primarily detected for fragments that are ejected along the laser polarization axis, the molecule is assumed to be aligned along the axis of the laser field. Further support for this assumption comes from the fact that aligned molecules are preferentially ionized via SFI. By inserting this Ansatz into the time-dependent Schrödinger equation one obtains the coupled equations

$$
i \frac{\partial}{\partial t}\left(\begin{array}{l}
\psi_{g}(R ; t) \\
\psi_{u}(R ; t)
\end{array}\right)=\left(\begin{array}{cc}
\frac{-1}{M} \frac{\partial^{2}}{\partial R^{2}}+V_{g}(R) & V_{g u}(R) \\
V_{g u}^{*}(R) & \frac{-1}{M} \frac{\partial^{2}}{\partial R^{2}}+V_{u}(R)
\end{array}\right)\left(\begin{array}{l}
\psi_{g}(R ; t) \\
\psi_{u}(R ; t)
\end{array}\right)
$$

with the binding potential curve $V_{g}(R)$, the dissociative curve $V_{u}(R)$ and the coupling between them $V_{g u}(R)$. Tabulated values for the potential curves were used [22]. Integration of eq. 5 yields the time-dependent nuclear wave functions. The initial condition directly after the recollision consists of placing the vibrational ground state, obtained by relaxation on the respective potential curve [23], onto the dissociative potential curve of the molecular ion $\left(2 p \sigma_{u+}\right)$. The recollision time for the first recollision is $1.7 \mathrm{fs}$ after ionization [32]. Due to the fact that the experiment employed few-cycle pulses, later recollision events are considered to be efficiently suppressed $[21,34]$. We note that the ionization is considered as a single event that occurs at the maximum of the laser electric field. This is a simplification, since the application of ADK theory [38] would predict that the ionization may occur during more than a single half-cycle of the laser, and furthermore, during a finite time interval within each half-cycle. A very rigorous theoretical treatment of the dynamics that goes well beyond the approach here and includes the ionization and recollision steps was recently presented by Gräfe and Ivanov [25]. However, a computational treatment of the hydrogen dissociation starting with an ADK treatment of the ionization is beyond the scope of the present paper, where our main aim is to qualitatively explain the physics responsible for the observed phase-control.

In the approach used here, for the calculation of the asymmetry, the electronic basis is changed to two states that are localized on the left and on the right nucleus, respectively. Without loss of generality, we define

$$
|l\rangle=\frac{1}{\sqrt{2}}(|g\rangle+|u\rangle)
$$

and

$$
|r\rangle=\frac{1}{\sqrt{2}}(|g\rangle-|u\rangle)
$$

By projecting onto these states, the corresponding nuclear wave functions are obtained. 
From these expressions the (t- as well as R-dependent) the probabilities for electron to remain on the left or on the right atom are calculated

$$
\begin{aligned}
& P_{l}(R, t)=\frac{1}{2}\left|\psi_{g}(R ; t)+\psi_{u}(R ; t)\right|^{2}, \\
& P_{r}(R, t)=\frac{1}{2}\left|\psi_{g}(R ; t)-\psi_{u}(R ; t)\right|^{2} .
\end{aligned}
$$

The time-dependent electron localization parameter is then defined as

$$
\frac{\int\left(P_{l}(R, t)-P_{r}(R, t)\right) d R}{\int\left(P_{l}(R, t)+P_{r}(R, t)\right) d R} \text {. }
$$

From these expressions we can immediately see that it is the coherent superposition of the two electronic states $1 s \sigma_{g+}$ and $2 p \sigma_{u+}$ that is responsible for the asymmetry in the charge localization.

In Figure 5, the temporal evolution of the laser field and the time-dependent electron localization parameter quantifying the localization on the upper/lower nucleus is displayed. The initial asymmetry that develops in the electron density is synchronized to the laser frequency, the intuitive picture being that the laser drives the electron back-and-forth (on attosecond timescales) between the two nuclei. However, as the molecule dissociates, the oscillatory motion of the electron between the two nuclei is impeded by the emergence of a potential barrier between the two nuclei. For an internuclear distance which is close to the internuclear distance where enhanced ionization would occur the electron oscillations stops and the electron density is found to localize predominantly on one of the atoms. In agreement with the experimental observation shifting the CEP by $\pi$ turns the laser field and thus the asymmetry around.

The observation of asymmetric $\mathrm{D}^{+}$emission as a result of electron localization requires that in our velocityresolved $\mathrm{D}^{+}$measurements we are unable to identify the quantum path (i.e. the $1 s \sigma_{g_{+}}$or the $2 p \sigma_{u+}$ curve) along which the measured ions were created. This restricts the kinetic energy range where an asymmetric emission may be expected. In good agreement with the experiment, the asymmetry is calculated to peak at around $6 \mathrm{eV}$. Importantly, no electron localization is observed at the very highest kinetic energies that occur in the experiment, since the wave packet that dissociates on the $1 s \sigma_{g+}$ curve is necessarily slower than the wave packet that dissociates on the repulsive $2 p \sigma_{u+}$ curve. In the intermediate energy range between 2 and $8 \mathrm{eV}$ the charge localization phase dependence exhibits, if at all, only weak substructures. This behavior can be easily understood from the electron rescattering process, which allows for access to a broad energetic range in molecular excitation during the sub-cycle rescattering event. The interpretation of the low KER regime is, however, more complicated. Simulations in [21] show a larger phase offset of $\sim \pi$ between BS and RCE. As 
suggested by Roudnev and Esry [39], asymmetries could be the result of pure interference between the $1 s \sigma_{g+}$ and $2 p \sigma_{u+}$ molecular channels populated directly via sequential optical excitation. If the nuclei are dissociating in two different molecular channels, they can still contribute at the same kinetic energy. In the few cycle case, laser bandwidth and Stark-shifting of the initial state may be strong enough to create this overlap of nuclear wavepackets, so that the relative phase between the final components still depends on the CEP.

Another quite interesting aspect comes into play from the $\theta$-dependence of the asymmetry as illustrated in Figures 2B and 4A. Within our simplified model, interference at low dissociation energies means that the low energy tail of the dissociative excited state wavepacket is interfering with a wavepacket that must have been stimulated from $2 p \sigma_{u+}$ to $1 s \sigma_{g+}$ by the laser field relatively early after the rescattering excitation process. We suggest that early de-excitation should be more dependent on the molecular alignment, which would explain that in Figure 4 the low energy region populates a smaller angular range. On the other hand, interference at high energy means that the downward transition takes place relatively late. When the gap between the two potential curves becomes less than a photon, it should suddenly become very difficult to induce a transition and only the very well aligned molecules may still succeed. Again, the angular selection becomes stricter, as observed in our experiment.

To conclude, within our modeling, we understand final charge localization during molecular dissociation in the following way. The molecular ions are formed in a (single) ionization event that occurs at the maximum of the laser electric field. The ionization event starts a vibrational wave packet in the $1 s \sigma_{g+}$ ground electronic state of $\mathrm{D}_{2}^{+}$that mimics the vibrational ground state wave function of $\mathrm{D}_{2}$ before excitation. Rescattering then leads to population transfer from the $1 s \sigma_{g}$ ground electronic state to the $2 p \sigma_{u+}$ excited electronic state at a delay of $\sim 1.7 \mathrm{fs}[32]$ after ionization. Because of the strongly repulsive nature of the $2 p \sigma_{u+}$ state, the excited $\mathrm{D}_{2}{ }^{+}$molecule rapidly dissociates and the resulting fragments acquire significant kinetic energies up to $10 \mathrm{eV}$. During the molecular dissociation the laser field can, however, transfer part of the $2 p \sigma_{u+}$ population back into the $1 s \sigma_{g+}$ state, thereby producing a dissociative wave packet with large excess kinetic energy. The emerging coherent superposition of the two electronic states results in a time-dependent localization of the electron density on the upper or lower nucleus due to the gerade and ungerade nature of the two states.

\section{$\underline{\text { Results II: electron localization in HD }}$}

Following the experimental demonstration of CEP control of electron localization in $\mathrm{D}_{2}$ further experiments were performed exploring the possibility to control electron localization in HD. Figure $6 \mathrm{~A}$ shows the $\mathrm{D}^{+}$ion kinetic energy spectrum that is obtained after excitation of HD with 5 fs laser pulses at $10^{14} \mathrm{~W} \mathrm{~cm}^{-2}$. Unlike the case of $\mathrm{D}_{2}$ the HD measurements were successfully accompanied by a measurement of above-threshold ionisation in Xe, allowing to assign a CEP of $\pi / 5$. The insert depicts a typical velocity map image, from which the energy spectrum has been obtained after angular integration. By varying the CEP an asymmetry map $A\left(W, \varphi_{\mathrm{CEP}}\right)$ was obtained (see Fig. $\left.6 \mathrm{~B}\right)$. In comparison to the homonuclear $\mathrm{D}_{2}$ case, quite similar asymmetries are obtained for the heteronuclear molecule. Figure 7A shows related data for $\mathrm{H}^{+}$from $\mathrm{HD}$ for the same excitation conditions ( $5 \mathrm{fs}, 10^{14} \mathrm{~W} \mathrm{~cm}^{-2}$ ). Note that the energy spectra differ by approximately a factor of $\sqrt{2}$ due to momentum conservation during the dissociation process. In general, proton spectra tend to show more noise, because of an increased background from ionization of $\mathrm{H}_{2} \mathrm{O}$. Apart from this additional noise, the $\mathrm{D}^{+}$and $\mathrm{H}^{+}$ion spectra shown in Figures 6 and 7 show very comparable asymmetry features. The asymmetries are also very similar to the homonuclear case displayed in Figure 3 . As seen in Figure $6 \mathrm{~B}$, for $\mathrm{D}^{+}$the asymmetry becomes prominent from 2 to $6 \mathrm{eV}$ and for $\mathrm{H}^{+}$shown in Figure 7B between 3 and $8 \mathrm{eV}$. Note that the phase features of $\mathrm{H}^{+}$and $\mathrm{D}^{+}$in figures 6 and 7 do not coincide in full, which is possibly due to the low 
signal-to-noise ratio in the $\mathrm{H}^{+}$measurements. As for $\mathrm{D}_{2}$, an asymmetry oscillation is also observed for $\mathrm{HD}$ at lower ion kinetic energies within the range of the bond softening contribution (0.7-1.5 and 1-2 eV for $\mathrm{D}^{+}$and $\mathrm{H}^{+}$, respectively). Again, a shift of the phase of the asymmetry oscillation between the low and high energy channels of approximately $\pi / 2$ is seen (see figures $6 \mathrm{C}$ and $7 \mathrm{C}$ ).

Figure 8 shows the energy and angular dependence of the amplitude $A_{0}(W, \theta)$ of the asymmetry oscillation $A\left(W, \theta, \varphi_{\mathrm{CEP}}\right)=A_{0}(W, \theta) \sin \left(\varphi_{\mathrm{CEP}}+\varphi_{0}(W, \theta)\right)$ that was obtained for $\mathrm{D}^{+}$ions from the dissociative ionization of HD. Similar to Figure $4 \mathrm{~B}$, where this analysis was performed for $\mathrm{D}_{2}$, the asymmetry is restricted to angles $\theta<50$ degree and shows a significant difference in the angular distribution of the asymmetry between the low $(0.7-1.5 \mathrm{eV})$ and high energy (above $2 \mathrm{eV}$ ) channels. The kinetic energy range where the asymmetries are observed for $\mathrm{H}^{+}$and $\mathrm{D}^{+}$from HD is lower than the kinetic range where these effects were observed for $\mathrm{D}_{2}$. A possible reason for this may be the fact that the vibrational period of $\mathrm{HD}$ is shorter than that of $\mathrm{D}_{2}$, meaning that the vibrational wave packet that is initially produced in the $1 s \sigma_{g}$ ground electronic state moves farther out during the 1.7 fsec separating the ionisation and the recollision event. If so, the recollision excitation promotes the nuclear wave packet to a somewhat lower position on the repulsive $2 p \sigma_{u}$ curve.

The CEP in Figures 6 and 7 has been determined in situ as an absolute phase via a reference measurement of the asymmetry $A\left(W, \varphi_{\text {CEP }}\right)$ in the electron emission in above-threshold ionization (ATI) of Xe (see Figure 9A for the ATI spectrum and 9B for the asymmetry map). The CEP was set to zero at positions where the cut-off electron emission reaches its maximum in the upward direction [40]. This should facilitate direct comparison to theoretical studies of the system. Interestingly, in HD, in agreement with recent theoretical findings [21], the emission of ions to one of the two sides of the laser polarization does not necessarily coincide with phase values of $\varphi_{\mathrm{CEP}}=n \cdot \pi($ with integer number $n)$.

The phase difference between the high-energy (recollision) and low-energy (bond softening) channels in both $\mathrm{D}_{2}$ and $\mathrm{HD}$ of $\sim \pi / 2$ is reminiscent to shifts that have been reported between direct and rescattered ATI photoelectron spectra for rare gas atoms, which for the direct (low energy) electrons have been explained as manifestation of double slits in time [14]. In the present experiment, however, the mechanism is slightly different. Based on inspection of the kinetic energy distributions in Figures 6 and 7 we have attributed the asymmetries at low energies to the onset of contributions from a (direct) bond softening (BS) channel, while higher energies have been attributed to (indirect) recollisional excitation (RCE). The difference in the ionization mechanism is therefore in principle similar to [14]. However, in the molecular case the closely coupled electron-nuclear dynamics has to be additionally taken into account. Thus, the asymmetry of the BS channel should rather be understood in terms of an n-photon pathway interference between the two respective trajectories for the dissociation of the Hydrogen molecular ion. During evolution of the molecule the laser field can couple the gerade and the ungerade states directly when the wavepacket approaches the outer potential well [26]. This coupling can be made responsible for the observed phase dependence as calculated by Roudnev and Esry for $\mathrm{HD}^{+}$[39]. Moreover, weak phase shifted asymmetries for total ion kinetic energies below $5 \mathrm{eV}$ have been observed by [21] in $\mathrm{D}_{2}$ model calculations, and have been attributed to asymmetric dissociation as described in [24].

\section{Conclusion}

Electron transfer processes play a pivotal role in chemistry. Presently, following the generation and measurement of single sub-femtosecond pulse made possible by unprecedented control of bound and free atomic electrons, respectively, with the sub-cycle evolution of a strong light field [41], it may be become 
possible to observe electron transfer processes on the fastest timescales that these processes take place. When attosecond pulses are used to initiate electron dynamics in molecules, the high photon energy of the attosecond pulse generally results in ionization. As discussed by Remacle and Levine [42], removal of an electron on attosecond timescales will often result in the formation of electronic wave packets, because the electron-hole density that results from removing an electron from the highest-occupied molecular orbital (HOMO) does not match the electron-hole density in the singly occupied HOMO of the cation formed on ionisation. Ultrafast removal of an electron therefore not only forms the ground electronic state of the cation, but, rather, a coherent superposition of electronic states. Remacle and Levine [42] have argued that the formation of this wave packet may lead to electron transport across the ionic structure that is formed. For example, the photoionization of the neutral tetrapeptide molecule TrpLeu 3 is expected to lead to the population of the HOMO-1 and the HOMO of the $\operatorname{TrpLeu}_{3}{ }^{+}$cation. The shape of these orbitals and the 3-eV energy splitting between the two orbitals suggest that electron transfer from one end of the molecule to the other occurs in less than 1 fs. Comparing a wide range of electronic systems, Breidbach and Cederbaum [43] observed that the sudden removal of an electron is accompanied by a characteristic time response completed in approximately 50 as. This time response is interpreted in terms of a filling - upon ionization - of the exchange-correlation hole associated with the electron ionized by its neighbouring electrons.

The time that sub-femtosecond pulses are used to initiate the formation and subsequently probe the formation of an electron wavepacket that transfers electron density across a large molecule has not yet come. However, in the present paper we have extended sub-femtosecond electron control to molecules and obtained first evidence of its usefulness in controlling reaction dynamics. We have controlled the dissociation of $\mathrm{D}_{2}^{+}$and $\mathrm{HD}^{+}$by steering electron wave packet motion with the sub-cycle, i.e. sub-femtosecond evolution of the electric field of a few-cycle light wave. A coherent superposition of two electronic states in the molecular ion is responsible for an oscillating electron density and the final localization of the electron. While the computed electron and nuclear dynamics are consistent with our measurement, deeper insight into the role of fieldcontrolled electron dynamics in driving chemical reactions will require time-resolved investigations [25, 44, 45]. The door to such studies is now open thanks to the availability of sub-femtosecond extreme-ultraviolet (XUV) pulses synchronized with the few-cycle control pulse [41]. Synthesized ultrawide-band (multi-color) waveforms (comprising near-infrared, visible and possibly ultraviolet light), which can now both be produced and measured may dramatically enhance the efficiency of steering reactions by creating electronic wave packets and subsequently driving them towards selected sites in complex molecular systems. Indeed, recent theoretical work suggests that circular electronic motion in ring-shaped molecules can be induced by controlled light-fields [46].

Electron transfer processes are extremely important in chemistry and biology. For example, rapid electron transfer can promote both damage and repair of DNA base-pairs. Our results for the intense-field dissociative ionization of $\mathrm{D}_{2}$ and HD constitute a first example of the control of intra-molecular electronic dynamics under the influence of the laser phase and thus provide a first clue that intra-molecular electron transfer processes may be controllable by light fields of controlled evolution.

\section{Acknowledgement}

We acknowledge contributions by Y. Ni, J.I. Khan, M. Schultze, T. Uphues, J. Rauschenberger, M. Uiberacker and M. Drescher to these studies. We thank the European Union for support by the Marie Curie Research Training Network XTRA, MRTN-CT-2003-505138, a Marie Curie Intra-European Fellowship, MEIF-CT-2003-500947, and a European Reintegration Grant. The research of M.F.K., C.S. and M.J.J.V. is 
part of the research program of the "Stichting voor Fundamenteel Onderzoek der Materie (FOM)", which has been financially supported by the "Nederlandse Organisatie voor Wetenschappelijk Onderzoek (NWO). M.F.K., I.Z. and S.Z. acknowledge support by the Max-Planck Society and by the German Science Foundation via the Emmy-Noether program. This work was partly supported by the Cluster of Excellence "Munich Center for Advanced Photonics" (MAP).

\section{Figures and Captions}

Figure 1: A) Schematic view of the velocity map imaging experiment. Laser pulses are CEP controlled using a pair of wedges. The beam is focused with a spherical mirror $(f=40 \mathrm{~cm})$ into the center of the ion optics, where it crosses a molecular beam. Resulting ions are extracted and analyzed using a dual microchannel and phosphor screen detector. A typical velocity map image from the detection of $\mathrm{D}^{+}$ions in the dissociation of $\mathrm{D}_{2}$ with 5 fs pulses at $10^{14} \mathrm{~W} \mathrm{~cm}^{-2}$ without phase stabilization is shown in $\mathrm{B}$ ).

Figure 2: A) $\mathrm{D}^{+}$kinetic energy spectrum from the interaction of $\mathrm{D}_{2}$ with $5 \mathrm{fs}$ linear and circular polarized laser pulses at $10^{14} \mathrm{~W} \mathrm{~cm}^{-2}$ without phase stabilization B) Angular distributions for $\mathrm{D}^{+}$from $\mathrm{D}_{2}$ within three energy windows corresponding to the BS (0-2eV), EI (2-3eV) and RCE (3-8eV) channels as measured for the conditions in A) for linear polarization. C) Schematic diagram showing the different dissociation pathways that yield $\mathrm{D}^{+}$ions from $\mathrm{D}_{2}$ via dissociation of the molecular ion via recollisional excitation (RCE) or coulomb explosion of $\mathrm{D}_{2}^{2+}$ in enhanced ionization (EI); in strong laser fields, bond softening (BS) may lead to dissociation of the molecular ion, where the avoided crossing between diabatic potentials that are dressed by the laser field (as an example, the $2 p \sigma_{u}{ }^{+}$potential dressed with (-1) photon is drawn as a dashed line) results in an energy gap that gives rise to dissociation from vibrational levels that were originally bound [35]. BS was studied in great detail for different light intensities and pulse durations [47]. Note that further channels playing a role at higher intensities than in the present studies are omitted in the scheme.

Figure 3: A) $\mathrm{D}^{+}$kinetic energy spectrum with 5 fs linear laser pulses at $10^{14} \mathrm{~W} \mathrm{~cm}{ }^{-2}$ without phase stabilization. B) Map of asymmetry parameter $A(W, \theta)$ as a function of the $\mathrm{D}^{+}$kinetic energy $W$ and the carrier envelope phase $\varphi_{\mathrm{CEP}}$ (measured over a range of $6 \pi$ with a step size of $0.1 \pi$ ). C) Asymmetry integrated over several energy ranges versus the CEP. D) Degree of alignment of D+ ions (represented by the expectation value of $\left.\cos ^{2}(\theta)\right)$ versus the CEP for the same energy intervals as in C).

Figure 4: A) Maximum degree of asymmetry $\mathrm{A}_{0}(W, \theta)$ in the emission of $\mathrm{D}^{+}$ions from the dissociative ionization of $\mathrm{D}_{2}$ as a function of the emission angle and energy. The phase dependent asymmetry oscillations have been fit to sine functions $A\left(W, \theta, \varphi_{\mathrm{CEP}}\right)=A_{0}(W, \theta) \sin \left(\varphi_{\mathrm{CEP}}+\varphi_{0}(W, \theta)\right)$ to obtain the parameter $\mathrm{A}_{0}(W, \theta)$. The effect is limited to $\theta= \pm 50^{\circ}$. The butterfly shape of the effect indicates two different mechanisms, based on RCE for high energies and BS for small ion energies (see text). B) Experimental dependence of the asymmetry modulation depth in the emission of $\mathrm{D}^{+}$ions between 3 and $8 \mathrm{eV}$ from dissociation of $\mathrm{D}_{2}$ on the laser pulse duration.

Figure 5: Results of the simulations: About 2/3 of an optical cycle after an electron has been liberated from the neutral molecule (accompanied by the production of a wave packet evolves along the ground ionic state $\left.1 \mathrm{~s} \sigma_{\mathrm{g}}{ }^{+}\right)$, this electron recollides with the parent and excites the part of the population that is relevant for the explanation of the experimental results to the $2 \mathrm{p \sigma}_{\mathrm{u}}{ }^{+}$state. A superposition of both the $1 \mathrm{~s} \sigma_{\mathrm{g}}{ }^{+}$and the $2 \mathrm{p} \sigma_{\mathrm{u}}{ }^{+}$ 
states is formed in the laser field by population transfer. This breaks the parity of the electronic wavefunction, and allows to control the final localization of the charge on the "left" and the "right" part of the molecule.

Figure 6: Asymmetry data obtained for the case of $\mathrm{HD}, \mathrm{D}^{+}$ions. A) Sample image and kinetic energy spectrum for $\mathrm{D}^{+}$ions from the dissociation of $\mathrm{HD}$ with $5 \mathrm{fs}, 10^{14} \mathrm{Wcm}^{-2}$ pulses. B) Map of the asymmetry parameter $A(W, \theta)$ as a function of the $\mathrm{D}^{+}$kinetic energy $W$ and phase $\varphi_{\text {CEP. }}$ C) Asymmetry parameter integrated over selected energy ranges (as indicated) versus the CEP.

Figure 7: Asymmetry data obtained for the case of $\mathrm{HD}, \mathrm{H}^{+}$ions. A) Sample image and kinetic energy spectrum for $\mathrm{H}^{+}$ions from the dissociation of $\mathrm{HD}$ with $5 \mathrm{fs}, 10^{14} \mathrm{Wcm}^{-2}$ pulses. B) Map of the asymmetry parameter $A(W, \theta)$ as a function of the $\mathrm{H}^{+}$kinetic energy $W$ and phase $\varphi_{\text {CEP. }}$ C) Asymmetry parameter integrated over selected energy ranges versus the CEP.

Figure 8: Maximum degree of asymmetry $\mathrm{A}_{0}(W, \theta)$ in the emission of $\mathrm{D}^{+}$ions from the dissociative ionization of $\mathrm{HD}$ as a function of the emission angle and energy. The phase dependent asymmetry oscillations have been fit to sine functions $A\left(W, \theta, \varphi_{\mathrm{CEP}}\right)=A_{0}(W, \theta) \sin \left(\varphi_{\mathrm{CEP}}+\varphi_{0}(W, \theta)\right)$ to obtain the parameter $\mathrm{A}_{0}(W, \theta)$.

Figure 9: A) Spectrum and B) asymmetry map $A\left(W, \varphi_{\mathrm{CEP}}\right)$ for the emission of electrons in above-threshold ionization of Xe with $5 \mathrm{fs}$ pulses at $10^{14} \mathrm{~W} \mathrm{~cm}^{-2}$. The phase was set to zero at the maximum asymmetry for cut-off electrons and used to calibrate the phase axis in figures 6 and 7.

\section{References}

1. Lenzner, M., et al., 1998, Extreme nonlinear optics with few-cycle laser pulses. IEICE Transactions, E81-C(2): p. 112-122.

2. Baltuska, A., et al., 2003, Attosecond control of electronic processes by intense light fields. Nature, 421(6923): p. 611-615.

3. Lewenstein, M., et al., 1994, Theory of High-Harmonic Generation by Low-Frequency Laser Fields. Phys. Rev. A, 49(3): p. 2117-2132.

4. Li, X.F., et al., 1989, Multiple-harmonic generation in rare gases at high laser intensity. Phys. Rev. A, 39(11): p. 5751-5761.

5. $\quad$ Freeman, R.R., et al., 1987, Above-threshold ionization with subpicosecond laser pulses. Phys. Rev. Lett., 59(10): p. 1092-1095.

6. Paulus, G.G., et al., 1994, Plateau in above threshold ionization spectra. Phys. Rev. Lett., 72(18): p. 2851-2854.

7. Spanner, M., et al., 2004, Reading diffraction images in strong field ionization of diatomic molecules. J. Phys. B, 37(12): p. L243-L250.

8. Itatani, J., et al., 2004, Tomographic imaging of molecular orbitals. Nature, 432(7019): p. 867-871.

9. Paulus, G.G., et al., 2003, Measurement of the phase of few-cycle laser pulses. Phys. Rev. Lett., 91(25): p. 253004.

10. Liu, X., et al., 2004, Nonsequential double ionization at the single-optical-cycle limit. Phys. Rev. Lett., 93(26): p. 263001.

11. Reichert, J., et al., 1999, Measuring the frequency of light with mode-locked lasers. Opt. Comm., 172(1-6): p. 59-68.

12. Goulielmakis, E., et al., 2004, Direct Measurement of Light Waves. Science, 305: p. 1267-1269. 
13. Kienberger, R., et al., 2004, Atomic transient recorder. Nature, 427(6977): p. 817-821.

14. Lindner, F., et al., 2005, Attosecond double-slit experiment. Phys. Rev. Lett., 95(4): p. 040401.

15. Kling, M.F., et al., 2006, Control of electron localization in molecular dissociation. Science, 312(5771): p. 246-248.

16. Sansone, G., et al., 2006, Isolated single-cycle attosecond pulses. Science, 314(5798): p. 443-446.

17. Uiberacker, M., et al., 2007, Attosecond real-time observation of electron tunnelling in atoms. Nature, 446(7136): p. 627-632.

18. Cavalieri, A.L., et al., 2007, Attosecond spectroscopy in condensed matter. Nature, 449: p. 1029-1032.

19. Posthumus, J.H., 2004, The dynamics of small molecules in intense laser fields. Rep. Progr. Phys., 67(5): p. 623-665.

20. Alnaser, A.S., et al., 2005, Simultaneous real-time tracking of wave packets evolving on two different potential curves in H-2(+) + and D-2(+). Phys. Rev. A, 72(3): p. 030702.

21. Tong, X.M. and C.D. Lin, 2007, Dynamics of Light-Field Control of Molecular Dissociation at the Few-Cycle Limit. Phys. Rev. Lett., 98: p. 123002.

22. Peek, J.M., 1965, Eigenparameters for the $1 \mathrm{~s} \sigma_{\mathrm{g}}$ and $2 \mathrm{p} \sigma_{\mathrm{u}}$ Orbitals of $\mathrm{H}_{2}^{+}$J. Chem. Phys., 43(9): p. 3004-3006.

23. Kolos, W., K. Szalewicz, and H.J. Monkhorst, 1986, New Born--Oppenheimer potential energy curve and vibrational energies for the electronic ground state of the hydrogen molecule. J. Chem. Phys., 84(6): p. 3278-3283.

24. Bandrauk, A.D., S. Chelkowski, and H.S. Nguyen, 2004, Attosecond localization of electrons in molecules. Int. J. Quant. Chem., 100(6): p. 834-844.

25. Gräfe, S. and M. Ivanov, 2007, Effective Fields in Laser-Driven Electron Recollision and Charge Localization. Phys. Rev. Lett., 99: p. 163603.

26. Haljan, P., M.Y. Ivanov, and P.B. Corkum, 1997, Laser control of electron localization in molecules and double quantum wells. Laser Phys., 7(3): p. 839-843.

27. Cavalieri, A.L., et al., 2007, Intense 1.5-cycle near infrared laser waveforms and their use for the generation of ultra-broadband soft-x-ray harmonic continua. New J. Phys., 9(7): p. 242.

28. Rauschenberger, J., et al., 2006, Carrier-envelope phase-stabilized amplifier system. Laser Phys. Lett., 3(1): p. 37-42.

29. Verhoef, A.J., et al., 2006, Few-cycle carrier envelope phase-dependent stereo detection of electrons. Opt. Lett., 31(23): p. 3520-3522.

30. Lepine, F., et al., 2004, Atomic photoionization processes under magnification. Phys. Rev. A, 70: p. 033417.

31. Vrakking, M.J.J., 2001, An iterative procedure for the inversion of two-dimensional ion/photoelectron imaging experiments. Rev. Sci. Instr., 72(11): p. 4084-4089.

32. Niikura, H., et al., 2002, Sub-laser-cycle electron pulses for probing molecular dynamics. Nature, 417(6892): p. 917-922.

33. Niikura, H., et al., 2003, Probing molecular dynamics with attosecond resolution using correlated wave packet pairs. Nature, 421(6925): p. 826-829.

34. Alnaser, A.S., et al., 2004, Routes to control of H-2 Coulomb explosion in few-cycle laser pulses. Phys. Rev. Lett., 93(18): p. 183202.

35. Bucksbaum, P.H., et al., 1990, Softening of the H2+ Molecular Bond in Intense Laser Fields. Phys. Rev. Lett., 64(16): p. 1883-1886.

36. Niikura, H., P.B. Corkum, and D.M. Villeneuve, 2003, Controlling vibrational wave packet motion with intense modulated laser fields. Phys. Rev. Lett., 90(20): p. 203601. 
37. Ergler, T., et al., 2006, Spatiotemporal imaging of ultrafast molecular motion: Collapse and revival of the D2+ nuclear wave packet. Phys. Rev. Lett., 97(19): p. 193001.

38. Ammosov, M.V., N.B. Delone, and V.P. Krainov, 1986, Sov. Phys. JETP, 64: p. 1191.

39. Roudnev, V. and B.D. Esry, 2007, $\mathrm{HD}^{+}$in a short strong laser pulse: Practical consideration of the observability of carrier-envelope phase effects, Phys. Rev. A, 76: p. 023403.

40. Kling, M.F., et al., 2007, Imaging of carrier-envelope phase effects in above-threshold ionization with intense few-cycle laser fields. New J. Phys., submitted.

41. Goulielmakis, E., et al., 2007, Attosecond control and measurement: Lightwave electronics. Science, 317(5839): p. 769-775.

42. Remacle, F. and R.D. Levine, 2006, An electronic time scale in chemistry. Proc. Nat. Acad. Sci. USA, 103(18): p. 6793-6798.

43. Breidbach, J. and L.S. Cederbaum, 2005, Universal attosecond response to the removal of an electron. Phys. Rev. Lett., 94: p. 033901.

44. Niikura, H., et al., 2005, Attosecond dynamics using sub-laser-cycle electron pulses. J. Mod. Opt., 52(2-3): p. 453-464.

45. Yudin, G.L., et al., 2005, Attosecond photoionization of coherently coupled electronic states. Phys. Rev. A, 72(5): p. 051401.

46. Barth, I. and J. Manz, 2006, Periodic Electron Circulation Induced by Circularly Polarized Laser Pulses: Quantum Model Simulations for Mg-porphyrin. Angew. Chem. Intern. Ed., 45: p. 2962-2965.

47. Sändig, K., H. Figger, and T.W. Hänsch, 2000, Dissociation Dynamics of H2+ in Intense Laser Fields: Investigation of Photofragments from Single Vibrational Levels. Phys. Rev. Lett., 85(23): p. 48764879 . 